Cahiers du MONDE RUSSE

\section{Cahiers du monde russe}

Russie - Empire russe - Union soviétique et États indépendants

55/1-2 | 2014

Fiscalité, justice et conflit politique en Russie, premier tiers du XVIIle siècle

\title{
ПЕТР I КАК ВЕРШИТЕЛЬ ПРАВОСУДИЯ
}

Pierre le Grand, justicier

Peter the Great as a dispenser of justice

Dmitrij O. Serov

\section{(2) OpenEdition}

Journals

Édition électronique

URL : http://journals.openedition.org/monderusse/7981

DOI : 10.4000/monderusse.7981

ISSN : $1777-5388$

Éditeur

Éditions de l'EHESS

Édition imprimée

Date de publication : 1 janvier 2014

Pagination : 13-30

ISBN : 9782713224409

ISSN : $1252-6576$

Référence électronique

Dmitrij O. Serov, « Петр I как вершитель правосудия », Cahiers du monde russe [Онлайн],

55/1-2 | 2014, Выложить онлайн 01 janvier 2017, Наводить справки в 01 mai 2019. URL : http:// journals.openedition.org/monderusse/7981 ; DOI : 10.4000/monderusse.7981

Ce document a été généré automatiquement le 1 mai 2019.

2011 


\section{ПЕТР I КАК ВЕРШИТЕЛЬ}

\section{ПРАВОСУДИЯ}

Pierre le Grand, justicier

Peter the Great as a dispenser of justice

Dmitrij 0. Serov

1 В ХРЕСТОМАТИЙНО ИЗВЕСТНОМ ТРАКТАТЕ « ПРАВДА ВОЛИ МОНАРШЕЙ ВО ОПРЕДЕЛЕНИИ НАСЛЕДНИКА ДЕРЖАВЫ СВОЕЙ», ВПЕРВЫЕ ОПУБЛИКОВАННОМ В 1722 Г., МЕЖДУ ИНОГО ЧИТАЕТСЯ ВЕСЬМА ПРИМЕЧАТЕЛЬНЫЙ ФРАГМЕНТ, В КОТОРОМ ИЗЛОЖЕНЫ ОБЯЗАННОСТИ ОБРАЗЦОВОГО ГОСУДАРЯ. УЧИТЫВАЯ ОФИЦИОЗНЫЙ ХАРАКТЕР ТРАКТАТА (АВТОРОМ КОТОРОГО ПРЕЖДЕ ОБЩЕПРИНЯТО БЫЛО СЧИТАТЬ ФЕОФАНА ПРОКОПОВИЧА) СЛЕДОВАЛО БЫ ОЖИДАТЬ, ЧТО ПЕРВЕЙШЕЙ СРЕДИ ЭТИХ ОБЯЗАННОСТЕЙ ОКАЖЕТСЯ ЗАБОТА О ВНЕШНЕЙ БЕЗОПАСНОСТИ ГОСУДАРСТВА ${ }^{1}$. ОДНАКО ПОПЕЧЕНИЕ О ВОИНСКОМ ДЕЛЕ ОКАЗАЛОСЬ, КАК НИ НЕОЖИДАННО, ПОМЕЩЕНО ЛИШЬ НА ВТОРОЕ МЕСТО : « ТАКОЖ И ДА БУДЕТ КРЕПКОЕ И ИСКУСНОЕ ВОИНСТВО НА ЗАЩИЩЕНИЕ ВСЕГО ОТЕЧЕСТВА ОТ НЕПРИЯТЕЛЯ»².

2 НА ПЕРВОЕ ЖЕ МЕСТО В РЯДУ ВЫСОЧАЙШИХ ОБЯЗАННОСТЕЙ СОСТАВИТЕЛЬ « ПРАВДЫ ВОЛИ МОНАРШЕЙ» ПОСТАВИЛ ЗАБОТУ МОНАРХА О ПРАВОСУДИИ: «ДА БУДУТ ЖЕ ПОДДАНИИ В БЕЗПЕЧАЛИИ, ДОЛЖЕН ЦАРЬ ПЕЩИСЯ ДА БУДЕТ ИСТИННОЕ В ГОСУДАРСТВЕ ПРАВОСУДИЕ НА ОХРАНЕНИЕ ОБИДИМЫХ ОТ ОБИДЯЩИХ» ПУБЛИКАЦИЮ ТРАКТАТА ПЕТР І ПОЛАГАЛ, ЧТО ЗАНЯТИЯ СУДОПРОИЗВОДСТВОМ БОЛЕЕ ВАЖНЫ, НЕЖЕЛИ ЗАНЯТИЯ ПО УСТРОЕНИЮ АРМИИ И СТОЛЬ ЛЮБЕЗНОГО ЕГО СЕРДЦУ ФЛОТА? ИЛИ ПРИВЕДЕННЫЕ СТРОКИ ЯВЛЯЛИСЬ ВСЕГО ЛИШЬ РИТОРИКОЙ, НЕ ОТРАЖАВШЕЙ НИ ПОДЛИННЫЕ ВОЗЗРЕНИЯ, НИ РЕАЛЬНЫЕ ПРИОРИТЕТЫ В ДЕЯТЕЛЬНОСТИ ПЕРВОГО РОССИЙСКОГО ИМПЕРАТОРА ?

3 ТЕМА ЛИЧНОГО УЧАСТИЯ ПЕТРА І В ОТПРАВЛЕНИИ ПРАВОСУДИЯ ДОНЫНЕ СПЕЦИАЛЬНО НЕ ОСВЕЩАЛАСЬ НИ ПРАВОВЕДАМИ, НИ ИСТОРИКАМИ. В ЧАСТНОСТИ, ОНА ОКАЗАЛАСЬ ПОЧТИ НИКАК НЕ ЗАТРОНУТА В ДВУХ СОВРЕМЕННЫХ МОНОГРАФИЯХ, АВТОРЫ КОТОРЫХ УГЛУБЛЕННО ОСВЕТИЛИ МНОГИЕ ПРОБЛЕМЫ КАК ФУНКЦИОНИРОВАНИЯ СУДЕБНОЙ 
СИСТЕМЫ, ТАК И РАЗВИТИЯ УГОЛОВНОГО И УГОЛОВНО-ПРОЦЕССУАЛЬНОГО ЗАКОНОДАТЕЛЬСТВА РОССИИ ПЕТРОВСКОГО ВРЕМЕНИ ${ }^{4}$. МЕЖДУ ТЕМ, БЕЗ ПРОЯСНЕНИЯ ОЗНАЧЕННОЙ ТЕМЫ НЕВОЗМОЖНО СИСТЕМАТИЧЕСКИ ПРЕДСТАВИТЬ, С ОДНОЙ СТОРОНЫ, ПРАВИТЕЛЬСТВЕННУЮ ДЕЯТЕЛЬНОСТЬ ПЕТРА І, А С ДРУГОЙ - ФУНКЦИОНИРОВАНИЕ ГОСУДАРСТВЕННОГО МЕХАНИЗМА РОССИИ В КОНЦЕ XVII - ПЕРВОЙ ЧЕТВЕРТИ ХVIII ВВ. ИТАК, КАКОЕ ЖЕ МЕСТО ЗАНИМАЛА СУДЕБНАЯ ПРАКТИКА В КРУГЕ ЗАНЯТИЙ ПЕРВОГО РОССИЙСКОГО ИМПЕРАТОРА? ПРЕЖДЕ ВСЕГО НЕОБХОДИМО ОТМЕТИТЬ, ЧТО В ЗАКОНОДАТЕЛЬСТВЕ КОНЦА ХVII ПЕРВОЙ ЧЕТВЕРТИ ХVIII ВВ. ПОРЯДОК НЕПОСРЕДСТВЕННОГО УЧАСТИЯ РОССИЙСКОГО МОНАРХА В СУДОПРОИЗВОДСТВЕ РЕГЛАМЕНТИРОВАЛСЯ ФРАГМЕНТАРНО ${ }^{5}$ ИСКЛЮЧЕНИЕМ ЯВЛЯЛОСЬ ЛИШЬ БОЛЕЕ ДЕТАЛЬНОЕ РЕГУЛИРОВАНИЕ ЕГО УЧАСТИЯ В ВОЕННО-УГОЛОВНОМ ПРОЦЕССЕ ${ }^{6}$. ЗДЕСЬ НЕОБХОДИМО ВСПОМНИТЬ ИМЕННОЙ УКАЗ ОТ 3 МАРТА 1719 Г., В КОТОРОМ УСТАНАВЛИВАЛОСЬ ОБЯЗАТЕЛЬНОЕ УТВЕРЖДЕНИЕ ЦАРЕМ СМЕРТНЫХ ПРИГОВОРОВ, ВЫНЕСЕННЫХ ВОЕННЫМИ СУДАМИ ЛИЦАМ, ИМЕВШИМ ОФИЦЕРСКОЕ ЗВАНИЕ 7 . ЭТОТ ПОРЯДОК БЫЛ ИЗМЕНЕН В САМОМ КОНЦЕ ПРАВЛЕНИЯ ПЕТРА І : СОГЛАСНО ВЫСОЧАЙШЕЙ РЕЗОЛЮЦИИ ОТ 11 НОЯБРЯ 1724 Г., НАЛОЖЕННОЙ НА ДОКЛАД ВОЕННОЙ КОЛЛЕГИИ, НА УТВЕРЖДЕНИЕ ИМПЕРАТОРА ДОЛЖНЫ БЫЛИ ВПРЕДЬ ПОСТУПАТЬ СМЕРТНЫЕ ПРИГОВОРЫ, ВЫНЕСЕННЫЕ ТОЛЬКО СТАРШИМ ОФИЦЕРАМ ${ }^{8}$. В ДАННОМ СЛУЧАЕ, С ФОРМАЛЬНО-ЮРИДИЧЕСКОЙ СТОРОНЫ МОНАРХ ВЫСТУПАЛ В КАЧЕСТВЕ ВЫСШЕЙ РЕВИЗИОННО-РЕШАЮЩЕЙ ИНСТАНЦИИ.

КРОМЕ ТОГО, НЕЛЬЗЯ НЕ ВСПОМНИТЬ И НАКАЗ « МАЙОРСКИМ» СЛЕДСТВЕННЫМ КАНЦЕЛЯРИЯМ ОТ 9 ДЕКАБРЯ 1717 Г., В КОТОРОМ ЗАКРЕПЛЯЛОСЬ САНКЦИОНИРОВАНИЕ ГЛАВОЙ ГОСУДАРСТВА ПРИМЕНЕНИЯ ПЫТОК В ОТНОШЕНИИ ПОДСЛЕДСТВЕННЫХ ИЗ ЧИСЛА ДОЛЖНОСТНЫХ ЛИЦ ГРАЖДАНСКОЙ АДМИНИСТРАЦИИ ОТ ВИЦЕ-ГУБЕРНАТОРА И ВЫШЕ, А ТАКЖЕ В ОТНОШЕНИИ СТАРШИХ И ВЫСШИХ ОФИЦЕРОВ 9 . НАЗНАЧЕНИЕ ПЫТОК ВЫСОКОПОСТАВЛЕННЫМ ЛИЦАМ ДОПУСКАЛОСЬ В РОССИИ ТОЛЬКО С РАЗРЕШЕНИЯ МОНАРХА, НЕСОМНЕННО, С ВЕСЬМА ДАВНИХ ВРЕМЕН, ОДНАКО ЛИШЬ В 1717 Г. ЭТА ПРОЦЕДУРА ВПЕРВЫЕ ОКАЗАЛАСЬ ПРОПИСАНА de jure. НАРЯДУ С ЭТИМ, ИМЕННЫМ УКАЗОМ ОТ 7 МАРТА 1721 Г. НА ОБЯЗАТЕЛЬНОЕ УТВЕРЖДЕНИЕ ЦАРЯ БЫЛО ПРЕДПИСАНО НАПРАВЛЯТЬ ПРИГОВОРЫ, ВЫНЕСЕННЫЕ СЕНАТОМ ПО ДЕЛАМ О КАЗНОКРАДСТВЕ ${ }^{10}$. В ИМЕННЫХ УКАЗАХ ОТ 17 МАРТА 1714 Г. И ОТ 19 ДЕКАБРЯ 1718 Г. ОБ УКРЕПЛЕНИИ ИНСТАНЦИОННОСТИ В СУДОПРОИЗВОДСТВЕ БЫЛО В ОБЩЕМ ВИДЕ ОБОЗНАЧЕНО ПОЛОЖЕНИЕ МОНАРХА КАК СУДА ЧЕТВЕРТОГО (ВЫСШЕГО) ЗВЕНА ${ }^{11}$.

НЕОБХОДИМО ОТМЕТИТЬ, ЧТО В СТ. 6 ИМЕННОГО УКАЗА ОТ 19 ДЕКАБРЯ 1718 Г. ДОПОЛНИТЕЛЬНО ОГОВАРИВАЛОСЬ, ЧТО НА РАССМОТРЕНИЕ ЦАРЯ МОГЛИ ПЕРЕДАВАТЬСЯ ИСКЛЮЧИТЕЛЬНО ТЕ СУДЕБНЫЕ ДЕЛА, РАЗРЕШЕНИЕ КОТОРЫХ ВЫЗВАЛО ЗАТРУДНЕНИЯ У ПРАВИТЕЛЬСТВУЮЩЕГО СЕНАТА. В СВОЮ ОЧЕРЕДЬ, СОГЛАСНО СТ. 4 ТОГО ЖЕ УКАЗА И СТ. 4 « ДОЛЖНОСТИ СЕНАТА» В РЕДАКЦИИ ОТ ДЕКАБРЯ 1718 Г., ДЕЛА МОГЛИ ПОСТУПАТЬ В СУДЕБНОЕ ПРОИЗВОДСТВО СЕНАТА ЛИШЬ ПО РАСПОРЯЖЕНИЮ МОНАРХА. СХОДНЫЙ ПОРЯДОК БЫЛ ЗАКРЕПЛЕН И В СТ. 4 « ДОЛЖНОСТИ СЕНАТА» В РЕДАКЦИИ ОТ 27 АПРЕЛЯ 1722 Г. (В КОТОРОЙ ПРОПИСЫВАЛАСЬ ТАКЖЕ ПРОЦЕДУРА ПЕРЕДАЧИ СООТВЕТСТВУЮЩИХ ВЫСОЧАЙШИХ УКАЗАНИЙ ЧЕРЕЗ ГЕНЕРАЛ-РЕКЕТМЕЙСТЕРА) ${ }^{12}$.

7 НАКОНЕЦ, НЕВОЗМОЖНО ОБОЙТИ УПОМИНАНИЕМ ПОДБОРКУ ЗАКОНОДАТЕЛЬНЫХ АКТОВ 1700-1718 ГГ., В КОТОРЫХ УСТАНАВЛИВАЛСЯ ОСОБЫЙ ПОРЯДОК СУДОПРОИЗВОДСТВА ПО ДЕЛАМ О ГОСУДАРСТВЕННЫХ ПРЕСТУПЛЕНИЯХ И О ПРЕСТУПЛЕНИЯХ ПРОТИВ ИНТЕРЕСОВ 
СЛУЖБЫ И ПРЕДУСМАТРИВАЛОСЬ ЛИЧНОЕ УЧАСТИЕ ЦАРЯ В ВОЗБУЖДЕНИИ ПОДОБНЫХ ДЕЛ. ПРИЧЕМ ЭТО ВЫСОЧАЙШЕЕ ВМЕШАТЕЛЬСТВО ОБУСЛАВЛИВАЛОСЬ НЕПОСРЕДСТВЕННЫМ ОБРАЩЕНИЕМ ПОДДАННЫХ.

СВОЕОБРАЗИЕ СИТУАЦИИ В ДАННОМ СЛУЧАЕ ЗАКЛЮЧАЛОСЬ В ТОМ, ЧТО, ХОТЯ ТРАДИЦИЯ ОБРАЩЕНИЯ ПОДДАННЫХ К МОНАРХУ (ПРАВО ЧЕЛОБИТЬЯ) СУЩЕСТВОВАЛА, КАК ИЗВЕСТНО, С ГЛУБОКОЙ ДРЕВНОСТИ, ЭТА ПРОЦЕДУРА ДЛИТЕЛЬНОЕ ВРЕМЯ НИКАК НЕ РЕГЛАМЕНТИРОВАЛАСЬ. ПЕРВАЯ ТАКОВАЯ РЕГЛАМЕНТАЦИЯ - В ВИДЕ ОГРАНИЧЕНИЯ ПРАВА ЧЕЛОБИТЬЯ - ПОСЛЕДОВАЛА В РОССИИ ЛИШЬ В СЕРЕДИНЕ XVII В., В СВЯЗИ С ВНЕСЕНИЕМ В СТ. 20 ГЛ. 10-Й УЛОЖЕНИЯ 1649 Г. НОРМЫ О ЗАПРЕТЕ ЧАСТНЫМ ЛИЦАМ ОБРАЩАТЬСЯ К ЦАРЮ, МИНУЯ ЦЕНТРАЛЬНЫЕ ОРГАНЫ (« В ПРИКАЗЕ НЕ БИВ ЧЕЛОМ»)13. В ПОСЛЕДУЮЩЕМ ЗАКОНОДАТЕЛЬСТВЕ XVII В. ДАННЫЙ ЗАПРЕТ НЕ ПОВТОРЯЛСЯ.

В ПЕРВОМ ИЗ ОЗНАЧЕННЫХ АКТОВ 1700-1718 ГГ. - ИМЕННОМ УКАЗЕ ОТ 2 ФЕВРАЛЯ 1700 Г.

- РЕЧЬ ШЛА О ПРАВЕ ПОДДАННОГО ИЗВЕЩАТЬ НЕПОСРЕДСТВЕННО ВЕРХОВНУЮ ВЛАСТЬ О

«ВЕЛИКИХ ГОСУДАРСТВЕННЫХ ДЕЛАХ» ${ }^{14}$. ПРИ ВСЕЙ НЕЧЕТКОСТИ ПРИВЕДЕННОЙ ФОРМУЛИРОВКИ, ВОЗМОЖНО С УВЕРЕННОСТЬЮ ПРЕДПОЛОЖИТЬ, ЧТО ПОД « ВЕЛИКИМИ ГОСУДАРСТВЕННЫМИ ДЕЛАМИ» ПЕТР I ПОДРАЗУМЕВАЛ В ДАННОМ СЛУЧАЕ КАК ПОПУЛЯРНЫЕ В ТЕ ГОДЫ ПРЕДЛОЖЕНИЯ ОБ УВЕЛИЧЕНИИ ГОСУДАРСТВЕННЫХ ДОХОДОВ, ТАК И СООБЩЕНИЯ ОБ ОСОБО ВАЖНЫХ ГОСУДАРСТВЕННЫХ ПРЕСТУПЛЕНИЯХ. ТЕМ САМЫМ, В ЗАКОНЕ ОТ 2 ФЕВРАЛСЯ 1700 Г. ВПЕРВЫЕ В ИСТОРИИ РОССИЙСКОГО ПРАВА ОКАЗАЛОСЬ ПРЯМО ЗАКРЕПЛЕНО (ХОТЯ И В РАСПЛЫВЧАТОЙ ФОРМЕ) ПРАВО ПОДДАННЫХ ОБРАЩАТЬСЯ НЕПОСРЕДСТВЕННО К МОНАРХУ С СООБЩЕНИЕМ О ПРЕСТУПЛЕНИИ ${ }^{15}$.

ДАННАЯ ЛИНИЯ НАШЛА ПРОДОЛЖЕНИЕ В ИМЕННОМ УКАЗЕ ОТ 23 ОКТЯБРЯ 1713 Г., СОБСТВЕННОРУЧНО НАПИСАННОМ ПЕТРОМ І. В ЭТОМ ЗАКОНЕ ЗАКРЕПЛЯЛОСЬ ПРАВО ЛЮБОГО ЖИТЕЛЯ СТРАНЫ - «ОТ ПЕРВЫХ ДАЖЕ ДО ЗЕМЛЕДЕЛЦОФ» - ИЗВЕЩАТЬ НЕПОСРЕДСТВЕННО МОНАРХА О «ГРАБИТЕЛЯХ НАРОДА» (ТО ЕСТЬ О ПРЕСТУПНОЙ ДЕЯТЕЛЬНОСТИ ДОЛЖНОСТНЫХ ЛИЦ ВСЕХ УРОВНЕЙ) ${ }^{16}$. В ИЗДАННОМ ДВА МЕСЯЦА СПУСТЯ ИМЕННОМ УКАЗЕ ОТ 23 ДЕКАБРЯ 1713 Г. ПОДДАННЫМ ДОЗВОЛЯЛОСЬ СООБЩАТЬ ЛИЧНО ЦАРЮ О ТАКИХ ПРЕСТУПЛЕНИЯХ КАК УМЫСЕЛ НА «ГОСУДАРСКОЕ ЗДОРОВЬЕ», ОСКОРБЛЕНИЕ « ВЫСОКОМОНАРШЕЙ ЧЕСТИ», БУНТ И ИЗМЕНА, ЧТО ЯВЛЯЛОСЬ ОЧЕВИДНОЙ КОНКРЕТИЗАЦИЕЙ ФОРМУЛИРОВКИ О «ВЕЛИКИХ ГОСУДАРСТВЕННЫХ ДЕЛАХ» ИЗ ИМЕННОГО УКАЗА ОТ 2 ФЕВРАЛЯ $1700 ~ Г .{ }^{17}$ СВОЕГО РОДА СИНТЕЗОМ ОТМЕЧЕННЫХ УКАЗОВ 1713 Г. ЯВИЛСЯ ИМЕННОЙ УКАЗ ОТ 25 ЯНВАРЯ 1715 Г. ЗДЕСЬ ПЕТР І СФОРМУЛИРОВАЛ ЗНАМЕНИТЫЕ « ТРИ ПУНКТА», СОДЕРЖАВШИЕ СОСТАВЫ ОСОБО ТЯЖКИХ ПРЕСТУПЛЕНИЙ, О ПОДГОТОВКЕ ИЛИ СОВЕРШЕНИИ КОТОРЫХ ТОЛЬКО И ДОПУСКАЛОСЬ НАПРЯМУЮ ИЗВЕЩАТЬ ВЕРХОВНУЮ ВЛАСТЬ. В ПЕРВЫХ ДВУХ ПУНКТАХ (ПОЧТИ В ПОЛНОМ СООТВЕТСТВИИ С ИМЕННЫМ УКАЗОМ ОТ 23 ДЕКАБРЯ 1713 Г.) ФИГУРИРОВАЛИ ГОСУДАРСТВЕННЫЕ ПРЕСТУПЛЕНИЯ : ПОСЯГАТЕЛЬСТВО НА ЖИЗНЬ САМОДЕРЖЦА, ИЗМЕНА И БУНТ. В ТРЕТЬЕМ ПУНКТЕ РЕЧЬ ШЛА О КАЗНОКРАДСТВЕ ${ }^{18}$. ПО СМЫСЛУ РАССМОТРЕННЫХ ЗАКОНОДАТЕЛЬНЫХ АКТОВ САМОДЕРЖЕЦ ВЫСТУПАЛ (В ОГОВОРЕННЫХ СЛУЧАЯХ) В РОЛИ ИНИЦИАТОРА УГОЛОВНОГО ПРЕСЛЕДОВАНИЯ.

11 ОСТАЕТСЯ ДОБАВИТЬ, ЧТО ВПЕРВЫЕ ЗАКРЕПЛЕННЫЙ В ИМЕННОМ УКАЗЕ ОТ 23 ОКТЯБРЯ 1713 Г. ОСОБЫЙ ПОРЯДОК СУДОПРОИЗВОДСТВА ПО ДЕЛАМ О ПРЕСТУПЛЕНИЯХ ПРОТИВ ИНТЕРЕСОВ СЛУЖБЫ СОХРАНЯЛСЯ ДО ИЗДАНИЯ ИМЕННОГО УКАЗА ОТ 19 ЯНВАРЯ 1718 Г., ПО КОТОРОМУ ИЗ ВЫШЕПРИВЕДЕННОГО СПИСКА 1715 Г. БЫЛ ПО СУЩЕСТВУ ИСКЛЮЧЕН ТРЕТИЙ ПУНКТ ${ }^{19}$. ОКОНЧАТЕЛЬНО ЖЕ ТРЕТИЙ ПУНКТ ИМЕННОГО УКАЗА ОТ 25 ЯНВАРЯ 
1715 Г. УТРАТИЛ СИЛУ В СВЯЗИ С ИЗДАНИЕМ ИМЕННОГО УКАЗА ОТ 19 ДЕКАБРЯ 1718 Г. ОБ УКРЕПЛЕНИИ ИНСТАНЦИОННОСТИ В СУДОПРОИЗВОДСТВЕ. СОГЛАСНО СТ. 7 НАЗВАННОГО УКАЗА, ЗАЯВЛЕНИЯ О КАЗНОКРАДСТВЕ НАДЛЕЖАЛО ПОДАВАТЬ ОРДИНАРНЫМ ПОРЯДКОМ В ФИСКАЛЬСКИЕ ОРГАНЫ ${ }^{20}$. МЕЧАТЕЛЬНО, ЧТО И В ПРОЕКТ УЛОЖЕНИЯ РОССИЙСКОГО ГОСУДАРСТВА 1723-1726 ГГ БЫЛО ВНЕСЕНО (В ПОДГОТОВЛЕННУЮ В ОКТЯБРЕ 1723 Г. СТАТЬЮ 42 ГЛ. 2-Й КН. 1) ЗАКОНОДАТЕЛЬНОЕ ПРЕДПОЛОЖЕНИЕ О ДОПУСТИМОСТИ ИЗВЕЩАТЬ НЕПОСРЕДСТВЕННО МОНАРХА ИМЕННО ПО ПЕРВЫМ ДВУМ ПУНКТАМ ИМЕННОГО УКАЗА ОТ 25 ЯНВАРЯ 1715 Г. В КАЧЕСТВЕ АЛЬТЕРНАТИВЫ ЗАЯВЛЕНИЕ ПО ЭТИМ ПУНКТАМ ПРЕДПИСЫВАЛОСЬ ПОДАВАТЬ В КАНЦЕЛЯРИЮ СЕНАТА ${ }^{21}$. РАССМОТРЕННЫМИ ЗАКОНОДАТЕЛЬНЫМИ АКТАМИ ИСЧЕРПЫВАЛАСЬ НОРМАТИВНАЯ ОСНОВА УЧАСТИЯ САМОДЕРЖЦА В РОССИЙСКОМ СУДОПРОИЗВОДСТВЕ КОНЦА XVII - ПЕРВОЙ ЧЕТВЕРТИ XVIII ВВ.

КАК ЖЕ СКЛАДЫВАЛОСЬ УЧАСТИЕ ПЕТРА І В СУДОПРОИЗВОДСТВЕ НА ПРАКТИКЕ ? ДЛЯ НАЧАЛА СЛЕДУЕТ ОТМЕТИТЬ, ЧТО, НЕСМОТРЯ НА ДЛИТЕЛЬНЫЕ ПОИСКИ В АРХИВНЫХ ФОНДАХ И ОПУБЛИКОВАННЫХ СБОРНИКАХ ДОКУМЕНТОВ, АВТОРУ НЕ УДАЛОСЬ К НАСТОЯЩЕМУ ВРЕМЕНИ ВЫЯВИТЬ НИ ЕДИНОГО ЭПИЗОДА УЧАСТИЯ ПЕРВОГО РОССИЙСКОГО ИМПЕРАТОРА В СУДЕБНОМ РАЗРЕШЕНИИ ГРАЖДАНСКИХ ДЕЛ.

ИЗВЕСТНО, ПРАВДА, ДВА СЛУЧАЯ, КОГДА ПРАВИТЕЛЬСТВУЮЩИЙ СЕНАТ НАПРАВЛЯЛ НА ВЫСОЧАЙШЕЕ РАССМОТРЕНИЕ ДЕЛА О ЖАЛОВАННОЙ ЧАСТИ НАСЛЕДСТВА СКОНЧАВШИХСЯ ВЫСШИХ ДОЛЖНОСТНЫХ ЛИЦ, НЕ ИМЕВШИХ НАСЛЕДНИКОВ ПЕРВОЙ ОЧЕРЕДИ. 31 МАРТА 1721 Г. ЦАРЮ БЫЛО ПРЕДСТАВЛЕНО ДЕЛО О СООТВЕТСТВУЮЩЕЙ ЧАСТИ НАСЛЕДСТВА ПОКОЙНОГО СЕНАТОРА Т.Н. СТРЕШНЕВА, А 22 СЕНТЯБРЯ ТОГО ЖЕ ГОДА - О НАСЛЕДСТВЕ ПОКОЙНОГО ПРЕЗИДЕНТА ВОЕННОЙ КОЛЛЕГИИ А.А. ВЕЙДЕ 22 ОДНАКО В ОБОИХ ЭПИЗОДАХ РЕЧЬ ШЛА, СТРОГО ГОВОРЯ, НЕ О ВЫНЕСЕНИИ СУДЕБНЫХ РЕШЕНИЙ (ПОСКОЛЬКУ ОТСУТСТВОВАЛ СУДЕБНЫЙ СПОР), А ОБ ОПРЕДЕЛЕНИИ ДАЛЬНЕЙШЕЙ СУДЬБЫ НАСЕЛЕННЫХ ИМЕНИЙ, РАНЕЕ ПОЖАЛОВАННЫХ НАЗВАННЫМ ЛИЦАМ ПЕТРОМ І.

НАПРОТИВ, К УГОЛОВНОМУ СУДОПРОИЗВОДСТВУ ПЕРВЫЙ РОССИЙСКИЙ ИМПЕРАТОР ИСПЫТЫВАЛ УСТОЙЧИВЫЙ ИНТЕРЕС НА ПРОТЯЖЕНИИ МНОГИХ ЛЕТ, НЕРЕДКО ПРИНИМАЯ УЧАСТИЕ КАК В СУДЕБНОМ, ТАК И В ДОСУДЕБНОМ ПРОИЗВОДСТВЕ ПО РАЗЛИЧНЫМ ДЕЛАМ. МОНАРХ-РЕФОРМАТОР ВОЗБУЖДАЛ УГОЛОВНОЕ ПРЕСЛЕДОВАНИЕ, ПРИНИМАЛ РЕШЕНИЯ О МЕРАХ ПРЕСЕЧЕНИЯ, ДОПРАШИВАЛ ОБВИНЯЕМЫХ И СВИДЕТЕЛЕЙ, САНКЦИОНИРОВАЛ РАЗЛИЧНЫЕ СЛЕДСТВЕННЫЕ ДЕЙСТВИЯ (ПРЕЖДЕ ВСЕГО, ПРИМЕНЕНИЕ ПЫТОК), ВЫНОСИЛ ИЛИ - ЧАЩЕ - УТВЕРЖДАЛ ПРИГОВОРЫ.

ЗАТРУДНИТЕЛЬНО В ТОЧНОСТИ СКАЗАТЬ, НАСКОЛЬКО ШИРОКО ОКАЗАЛИСЬ РЕАЛИЗОВАНЫ НА ПРАКТИКЕ НОРМЫ ЗАКОНОДАТЕЛЬНЫХ АКТОВ 1700-1718 ГГ. ОБ ОСОБОМ ПОРЯДКЕ СУДОПРОИЗВОДСТВА ПО ДЕЛАМ О ГОСУДАРСТВЕННЫХ ПРЕСТУПЛЕНИЯХ И О ПРЕСТУПЛЕНИЯХ ПРОТИВ ИНТЕРЕСОВ СЛУЖБЫ. СТАТИСТИКА ПОДОБНОГО РОДА В ТЕ ВРЕМЕНА НЕ ВЕЛАСЬ. ПО ВСЕЙ ВИДИМОСТИ, СОСРЕДОТОЧЕННОМУ НА РЕШЕНИИ ВОЕННЫХ, ДИПЛОМАТИЧЕСКИХ И ФИНАНСОВЫХ ВОПРОСОВ, НЕРЕДКО БОЛЕВШЕМУ ПЕТРУ І БЫЛО ЧАЩЕ ВСЕГО НЕДОСУГ ЛИЧНО ВЫСЛУШИВАТЬ ЗАЯВЛЕНИЯ ПОДДАННЫХ О ГОСУДАРСТВЕННЫХ И ДОЛЖНОСТНЫХ ПРЕСТУПЛЕНИЯХ.

ОДНИМ ИЗ ЯВСТВЕННО НЕМНОГИХ ПРИМЕРОВ РЕАЛИЗАЦИИ НА ПРАКТИКЕ НОРМ ИМЕННЫХ УКАЗОВ ОТ 25 ЯНВАРЯ 1715 Г. И ОТ 19 ДЕКАБРЯ 1718 Г. ОБ ОСОБОМ ПОРЯДКЕ СУДОПРОИЗВОДСТВА ПО ГОСУДАРСТВЕННЫМ ПРЕСТУПЛЕНИЯМ ЯВИЛСЯ ЭПИЗОД С ХОЛОПОМ ПРАПОРЩИКА Т.С. СКОБЕЕВА АКИМОМ ИВАНОВЫМ. ПОДСЛУШАВ ОТКРОВЕНИЯ 
ХОЗЯИНА ОБ ОСОБЕННОСТЯХ БЫТОВОГО ПОВЕДЕНИЯ ПЕТРА І, ХОЛОП ПРИШЕЛ 17 АПРЕЛЯ 1721 Г. В ЦАРСКУЮ РЕЗИДЕНЦИЮ В СЕЛЕ ПРЕОБРАЖЕНСКОМ, ГДЕ ОБЪЯВИЛ « СЛОВО И ДЕЛО» КАРАУЛЬНОМУ ОФИЦЕРУ. ИЗВЕТЧИКА НЕЗАМЕДЛИТЕЛЬНО ПРЕПРОВОДИЛИ К ЦАРЮ. И ХОТЯ ПЕТР І РЕЗОННО СЧЕЛ ИЗВЕТ НЕВАЖНЫМ», ОН НАПРАВИЛ ДЕЛО НА РАССМОТРЕНИЕ В ТАЙНУЮ КАНЦЕЛЯРИЮ, КОТОРАЯ И ПРИГОВОРИЛА 1 МАЯ 1722 Г. ИЗЛИШНЕ БОЛТЛИВОГО ТИМОФЕЯ СКОБЕЕВА К ТЕЛЕСНОМУ НАКАЗАНИЮ ${ }^{23}$. ОСОБЫЙ ПОРЯДОК СУДОПРОИЗВОДСТВА ПО ДЕЛАМ О ПРЕСТУПЛЕНИЯХ ПРОТИВ ИНТЕРЕСОВ СЛУЖБЫ НА ПРАКТИКЕ ОКАЗАЛСЯ, ПО ВСЕЙ ВИДИМОСТИ, ЕЩЕ МЕНЕЕ ЭФФЕКТИВНЫМ. В УСЛОВИЯХ ВТОРОГО ДЕСЯТИЛЕТИЯ XVIII В. БЫЛО, КОНЕЧНО, НЕРЕАЛЬНО ОБЕСПЕЧИТЬ БЫВШЕЕ ВОЗМОЖНЫМ РАЗВЕ ЧТО В УДЕЛЬНЫЕ ВРЕМЕНА - ШИРОКОЕ ЛИЧНОЕ УЧАСТИЕ ГЛАВЫ ГОСУДАРСТВА В РАЗБИРАТЕЛЬСТВЕ ДЕЛ О ПРОТИВОПРАВНОЙ ДЕЯТЕЛЬНОСТИ ПРЕДСТАВИТЕЛЕЙ ВЛАСТИ. ДУМАЕТСЯ, ИМЕННО ВОСПОМИНАНИЯ О СОБСТВЕННЫХ ПОПЫТКАХ ЗАНЯТЬСЯ РАЗБИРАТЕЛЬСТВОМ ДОНОШЕНИЙ О «ГРАБИТЕЛЯХ НАРОДА», ПРЕДПРИНЯТЫХ В СЕРЕДИНЕ 1710-ХГГ., ПОБУДИЛИ ПЕТРА І ЭМОЦИОНАЛЬНО ВЫСКАЗАТЬСЯ В СОБСТВЕННОРУЧНО НАПИСАННОЙ ПРЕАМБУЛЕ К ИМЕННОМУ УКАЗУ ОТ 19 ДЕКАБРЯ 1718 Г. О ТОМ, ЧТО « КОМУ БЬЮТ ЧЕЛОМ [ЦАРЮ], ОДНА ПЕРСОНА ЕСТЬ, И ТА КОЛИКИМИ ВОИНСКИМИ И ПРОТЧИМИ НЕСНОСНЫМИ ТРУДАМИ ОБЪЯТА... И ХОТЯ Б И ТАКИХ ТРУДОВ НЕ БЫЛО, ВОЗМОЖНО ЛЬ ОДНОМУ ЧЕЛОВЕКУ ЗА ТАК МНОГИМИ УСМОТРИТЬ, ВОИСТИННУ НЕ ТОЧНО ЧЕЛОВЕКУ, НИЖЕ АНГЕЛУ, ПОНЕЖЕ... ГДЕ ПРИСУТСТВУЕТ, ИНДЕ ЕГО НЕТ» ${ }^{24}$.

СРЕДИ СУДЕБНЫХ ДЕЛ, ОСОБЕННОЕ ВНИМАНИЕ МОНАРХА-РЕФОРМАТОРА ПРИВЛЕКАЛИ, ВО-ПЕРВЫХ, ДЕЛА ПО ГОСУДАРСТВЕННЫМ ПРЕСТУПЛЕНИЯМ, А ВО-ВТОРЫХ, ДЕЛА ПО ОБВИНЕНИЯМ ВЫСОКОПОСТАВЛЕННЫХ ДОЛЖНОСТНЫХ ЛИЦ В ПРЕСТУПЛЕНИЯХ ПРОТИВ ИНТЕРЕСОВ СЛУЖБЫ. ПРИМЕРОВ РАЗНООБРАЗНОГО УЧАСТИЯ ПЕТРА І В РАЗБИРАТЕЛЬСТВЕ ДЕЛ ПО ГОСУДАРСТВЕННЫМ ПРЕСТУПЛЕНИЯМ ИЗВЕСТНО МНОЖЕСТВО - ДОСТАТОЧНО ОБРАТИТЬСЯ К ТРУДАМ В.И. ВЕРЕТЕННИКОВА, Н.Б. ГОЛИКОВОЙ И Е.В. АНИСИМОВА ${ }^{25}$. ИЗ ОБШИРНОЙ ЧЕРЕДЫ ТАКИХ ДЕЛ БОЛЕЕ ВСЕГО ВРЕМЕНИ ПЕТР І УДЕЛИЛ (ЧТО ЛЕГКО ПОНЯТЬ) ПРОЦЕССУ ЦАРЕВИЧА АЛЕКСЕЯ ПЕТРОВИЧА. ЗДЕСЬ ЦАРЬ ПРИНЯЛ САМОЕ АКТИВНОЕ УЧАСТИЕ КАК В ПРЕДВАРИТЕЛЬНОМ СЛЕДСТВИИ (ОСУЩЕСТВЛЯВШИМСЯ СПЕЦИАЛЬНО УЧРЕЖДЕННОЙ КАНЦЕЛЯРИЕЙ ПОД РУКОВОДСТВОМ П.А. ТОЛСТОГО), ТАК И В СУДЕБНОМ ПРОИЗВОДСТВЕ ПО ДЕЛУ (КОТОРОЕ ВЕЛОСЬ ОСОБЫМ СУДЕБНЫМ ПРИСУТСТВИЕМ $)^{26}$. К ПРИМЕРУ, КОГДА ПОСТУПИЛИ ДАННЫЕ О ПОСОБНИЧЕСТВЕ ЦАРЕВИЧУ СО СТОРОНЫ ГЕНЕРАЛ-ЛЕЙТЕНАНТА КНЯЗЯ В.В. ДОЛГОРУКОВА, ПЕТР І НЕЗАМЕДЛИТЕЛЬНО ВОЗБУДИЛ ЕГО УГОЛОВНОЕ ПРЕСЛЕДОВАНИЕ И САНКЦИОНИРОВАЛ АРЕСТ (ПРОИЗВЕДЕННЫЙ $\quad$ В $\quad$ САНКТ-ПЕТЕРБУРГЕ 20 ФЕВРАЛЯ 1718 Г. ЛИЧНО ГЕНЕРАЛ-ГУБЕРНАТОРОМ И ПРЕЗИДЕНТОМ ВОЕННОЙ КОЛЛЕГИИ А.Д. МЕНШИКОВЫМ). ВСЛЕД ЗА ЭТИМ, 9 МАРТА 1718 Г., БУДУЩИЙ ИМПЕРАТОР ДОПРОСИЛ ДОСТАВЛЕННОГО В СЕЛО ПРЕОБРАЖЕНСКОЕ ПОДСЛЕДСТВЕННОГО. НАКОНЕЦ, ПОСЛЕ ТОГО КАК ОСОБОЕ СУДЕБНОЕ ПРИСУТСТВИЕ 14 МАРТА 1718 Г. ПРИГОВОРИЛО ВАСИЛИЯ ДОЛГОРУКОВА К ЛИШЕНИЮ ЧИНОВ, КОНФИСКАЦИИ ИМУЩЕСТВА И ССЫЛКЕ, ЦАРЬ 5 ИЮЛЯ 1718 Г. УТВЕРДИЛ ПРИГОВОР, ИЗБРАВ ОСУЖДЕННОМУ В КАЧЕСТВЕ МЕСТА ССЫЛКИ ГОРОД СОЛИКАМСК ${ }^{27}$

21 ЕСЛИ ГОВОРИТЬ О ДЕЛАХ ПО ПРЕСТУПЛЕНИЯМ ПРОТИВ ИНТЕРЕСОВ СЛУЖБЫ, ТО НЕИЗМЕННЫМ ВНИМАНИЕМ ПЕТРА І ПОЛЬЗОВАЛИСЬ ТЕ ИЗ НИХ, КОТОРЫЕ НАХОДИЛИСЬ В ПРОИЗВОДСТВЕ ПОДЧИНЕННЫХ НЕПОСРЕДСТВЕННО ЕМУ МАЙОРСКИХ» СЛЕДСТВЕННЫХ 
КАНЦЕЛЯРИЙ. ДОСТАТОЧНО СКАЗАТЬ, ЧТО ТОЛЬКО ГЛАВЕ ПЕРВОЙ ИЗ ТАКИХ КАНЦЕЛЯРИЙ ГВАРДИИ МАЙОРУ КНЯЗЮ М.И. ВОЛКОНСКОМУ ЦАРЬ НАПРАВИЛ В НОЯБРЕ 1713 - МАРТЕ 1715 ГГ. ШЕСТЬ ПИСЕМ, СОДЕРЖАВШИХ УКАЗАНИЯ ПО ПРОВЕДЕНИЮ СЛЕДСТВИЯ ПО « АРХАНГЕЛЬСКОМУ ДЕЛУ» ${ }^{28}$.

РАСПРЕДЕЛЕНИЕ УГОЛОВНЫХ ДЕЛ ПО МАЙОРСКИМ» КАНЦЕЛЯРИЯМ ПЕТР I ОСУЩЕСТВЛЯЛ ТАКЖЕ САМОЛИЧНО. К ПРИМЕРУ, 9 ДЕКАБРЯ 1717 Г. БУДУЩИЙ ИМПЕРАТОР НАПРАВИЛ В ПРОИЗВОДСТВО СЛЕДСТВЕННОЙ КАНЦЕЛЯРИИ Г.И. КОШЕЛЕВА И Ф.Д. ВОРОНОВА ВОЗБУЖДЕННЫЕ ФИСКАЛЬСКОЙ СЛУЖБОЙ ДЕЛА ПО ОБВИНЕНИЯМ ГВАРДИИ КАПИТАНА КНЯЗЯ А.Л. ДОЛГОРУКОВА, АРХАНГЕЛОГОРОДСКОГО ВИЦЕ-ГУБЕРНАТОРА П.Е. ЛОДЫЖЕНСКОГО, АРХАНГЕЛОГОРОДСКОГО ОБЕР-КОМИССАРА Д.А. СОЛОВЬЕВА, БЫВШЕГО АРХАНГЕЛОГОРОДСКОГО ВИЦЕ-ГУБЕРНАТОРА А.А. КУРБАТОВА, РИЖСКОГО ГУБЕРНАТОРА КНЯЗЯ П.А. ГОЛИЦЫНА, СЕНАТОРА М.М. САМАРИНА, КОМИССАРА П.И. ВЛАСОВА И ДЬЯКА П.К. СКУРИХИНА ${ }^{29}$. СОХРАНИЛОСЬ УНИКАЛЬНОЕ СВИДЕТЕЛЬСТВО О РЕАКЦИИ СОВРЕМЕННИКОВ НА УЧАСТИЕ ПЕТРА І В ПРОВОДИВШЕМСЯ СЛЕДСТВЕННОЙ КАНЦЕЛЯРИЕЙ В.В. ДОЛГОРУКОВА В 17141715 ГГ. ПРЕДВАРИТЕЛЬНОМ СЛЕДСТВИИ ПО ВОЗБУЖДЕННОМУ ФИСКАЛЬСКОЙ СЛУЖБОЙ «ПОДРЯДНОМУ ДЕЛУ», В КОТОРОМ ОКАЗАЛАСЬ ЗАМЕШАНА ГРУППА ВЫСШИХ ДОЛЖНОСТНЫХ ЛИЦ. ВОТ ЧТО ПИСАЛ НА ИСХОДЕ 1714 Г. НАХОДИВШЕМУСЯ В ГОЛЛАНДИИ БРАТУ ОСИПУ ВЕСЬМА ОСВЕДОМЛЕННЫЙ ДВОРЕЦКИЙ А.Д. МЕНШИКОВА Ф.А. СОЛОВЬЕВ : «СВЕТЛЕЙШИЙ КНЯЗЬ [А.Д. МЕНШИКОВ] В ВЕЛИКОЙ КОНФУЗИИ, И ВСЕ В САМОМ ПЕЧАЛНОМ ОБРАЗЕ, ПОНЕЖЕ ЦАРСКОЕ ВЕЛИЧЕСТВО ЗЕЛО ПРИЛЕЖНО СИЕ ДЕЛО [ПОДРЯДНОЕ] САМ РОЗЫСКИВАЕТ И НЕ ТОКМО СИЕ, НО И ВСЯКИЯ ДЕЛА САМ ОБЕЩАЕТСЯ ПЕРЕСМОТРЕТЬ И НАКАЗАТЬ КАК ЗА БОЛШОЕ, ТАК И ЗА МАЛОЕ РАВНО, О ЧЕМ ВСЕ ТРЯСУТСЯ...» ${ }^{30}$. «ТРЯСТИСЬ», ВПРОЧЕМ, БЫЛО ОТ ЧЕГО. ВПЕЧАТЛЕННЫЙ МАСШТАБАМИ ВСКРЫВШИХСЯ ФАЛЬШИВЫХ ПОДРЯДОВ, ПЕТР І РАСПОРЯДИЛСЯ ПЫТАТЬ САНОВНЫХ ФИГУРАНТОВ ДЕЛА. В ПРИСУТСТВИИ ЦАРЯ 27 НОЯБРЯ И 13 ДЕКАБРЯ 1714 Г. ПОДНИМАЛИ НА ДЫБУ СЕНАТОРА Г.И. ВОЛКОНСКОГО, 27 НОЯБРЯ - САНКТ-ПЕТЕРБУРГСКОГО ВИЦЕ-ГУБЕРНАТОРА Я.Н. РИМСКОГО-КОРСАКОВА ${ }^{31}$.

НЕ МЕНЬШЕ ВНИМАНИЯ ПЕТР I УДЕЛИЛ И МНОГИМ ДРУГИМ ДЕЛАМ ПО ОБВИНЕНИЮ ВЫСОКОПОСТАВЛЕННЫХ ЛИЦ В «ПРЕСТУПЛЕНИЯХ ДОЛЖНОСТИ СВОЕЙ». ТАК, МОНАРХ ПРИНЯЛ САМОЕ АКТИВНОЕ УЧАСТИЕ В ПРОЦЕССЕ ПО ДЕЛУ СЕНАТОРА И ВИЦЕ-ПРЕЗИДЕНТА КОЛЛЕГИИ ИНОСТРАННЫХ ДЕЛ БАРОНА П.П. ШАФИРОВА И ОБЕР-ПРОКУРОРА СЕНАТА Г.Г. СКОРНЯКОВА-ПИСАРЕВА. ИМПЕРАТОР СЫГРАЛ РЕШАЮЩУЮ РОЛЬ НА ВСЕХ СТАДИЯХ НАЗВАННОГО ПРОЦЕССА: ОТ ВОЗБУЖДЕНИЯ 9 ЯНВАРЯ 1723 Г. В ОТНОШЕНИИ ПЕТРА ШАФИРОВА И ГРИГОРИЯ СКОРНЯКОВА-ПИСАРЕВА УГОЛОВНОГО ПРЕСЛЕДОВАНИЯ ДО УТВЕРЖДЕНИЯ ВЫНЕСЕННЫХ ИМ 13 ФЕВРАЛЯ 1723 Г. ПРИГОВОРОВ ${ }^{32}$. УЧАСТВУЯ В ПРОЦЕССЕ, ПЕТР І ДОСКОНАЛЬНО ВНИКАЛ ДАЖЕ В ПРЯМО НЕ ОТНОСИВШИЕСЯ К ДЕЛУ ЭПИЗОДЫ, КОТОРЫЕ ВСКРЫВАЛИСЬ ПО ХОДУ ДОПРОСОВ ПОДСУДИМЫХ И СВИДЕТЕЛЕЙ. К ПРИМЕРУ, ПРОВЕРИВ ФИГУРИРОВАВШИЕ В ПОКАЗАНИЯХ Г.Г. СКОРНЯКОВА-ПИСАРЕВА СВЕДЕНИЯ О ПОДОЗРИТЕЛЬНЫХ ОБСТОЯТЕЛЬСТВАХ НАЗНАЧЕНИЯ НА ДОЛЖНОСТЬ ВИЦЕ-ПРЕЗИДЕНТА ЯРОСЛАВСКОГО НАДВОРНОГО СУДА КНЯЗЯ А.М. ШЕЙДЯКОВА, ИМПЕРАТОР РАСПОРЯДИЛСЯ НЕ ПРИНИМАТЬ ИХ ВО ВНИМАНИЕ, НАЛОЖИВ ПРОСТРАННУЮ РЕЗОЛЮЦИЮ : « ОТСТАВИТЬ, ДЛЯ ТОГО ЧТО ДОНОСИЛ, ЧТО ОН ЗА ПРОСТОТОЮ СВОЕЮ НЕ ГОДЕН, А ПРЕСТУПЛЕНИЯ ОН ЗА НИМ НИКАКОГО НЕ ЗНАЕТ ${ }^{33}$. 
МНОГО ВНИМАНИЯ УДЕЛИЛ ПЕТР І И НАЧАВШЕМУСЯ В 1722 Г. ПРОЦЕССУ НАД ОБЕР-ФИСКАЛОМ А.Я. НЕСТЕРОВЫМ. В ЧАСТНОСТИ, ИМПЕРАТОР ПРИНЯЛ УЧАСТИЕ ВО ВСЕХ ТРЕХ ДОПРОСАХ АЛЕКСЕЯ НЕСТЕРОВА ПОД ПЫТКОЙ : 30 ЯНВАРЯ 1723 Г., 16 И 18 ЯНВАРЯ $1724 \Gamma^{34} 29$ ЯНВАРЯ 1723 Г. ПЕТР І ДАЖЕ СОБСТВЕННОРУЧНО НАПИСАЛ ВОПРОСЫ К ПРЕДСТОЯВШЕМУ ДОПРОСУ ОБЕР-ФИСКАЛА. ВОПРОСЫ ЭТИ ПРЕДВАРЯЛИСЬ ЭМОЦИОНАЛЬНЫМ РАССУЖДЕНИЕМ О ТОМ, ЧТО «НИКОМУ ТАК О ТОМ [ПРЕСТУПНОЙ ДЕЯТЕЛЬНОСТИ ИНЫХ ДОЛЖНОСТНЫХ ЛИЦ] СВЕДОМОМУ БЫТЬ НЕВОЗМОЖНО КАК ТЕБЕ : ПЕРВОЕ, ПО ЧИНУ ФИСКАЛСКОМУ, ВТОРОЕ, ПОНЕЖЕ ПРИБЕЖИЩЕ ВСЕХ ВОРОВ БЫЛ...» ${ }^{35}$.

НА СЕГОДНЯ ИЗВЕСТЕН ЕДИНСТВЕННЫЙ СЛУЧАЙ, КОГДА ПЕРВЫЙ РОССИЙСКИЙ ИМПЕРАТОР ПРЕКРАТИЛ (ПО НЕПОНЯТНОЙ ПРИЧИНЕ) УГОЛОВНОЕ ПРЕСЛЕДОВАНИЕ ВЫСОКОПОСТАВЛЕННОГО ДОЛЖНОСТНОГО ЛИЦА. ЭТИМ ЛИЦОМ БЫЛ СТАРЕЙШИЙ СЕНАТОР, ГЕНЕРАЛ-ПЛЕНИПОТЕНЦИАР-КРИГСКОМИССАР КНЯЗЬ Я.Ф. ДОЛГОРУКОВ. ПО УСПЕШНО РАССЛЕДОВАННОМУ КАНЦЕЛЯРИЕЙ И.И. ДМИТРИЕВА-МАМОНОВА ЭПИЗОДУ О НЕЗАКОННОМ ЗАИМСТВОВАНИИ ЯКОВОМ ДОЛГОРУКОВЫМ 50 ТЫСЯЧ РУБЛЕЙ ИЗ КАССЫ ВОЕННОЙ КАНЦЕЛЯРИИ ДЛЯ КОММЕРЧЕСКОЙ «ПРОКРУТКИ», СЕНАТОР БЫЛ В 1718 Г. ПРЕДАН ГЕНЕРАЛЬНОМУ ВОЕННОМУ СУДУ ПОД ПРЕДСЕДАТЕЛЬСТВОМ А.А.ВЕЙДЕ. ПРИГОВОР ПО ЭТОМУ ДЕЛУ НЕ СОСТОЯЛСЯ, ПОСКОЛЬКУ ПЕТР І НЕОЖИДАННО ОСТАНОВИЛ СУДЕБНЫЙ ПРОЦЕСС ${ }^{36}$.

27 В ИТОГЕ, ПОДОЗРЕВАВШИЙСЯ В МНОГОКРАТНОМ ПОЛУЧЕНИИ ВЗЯТОК И ЗЛОУПОТРЕБЛЕНИИ ДОЛЖНОСТНЫМИ ПОЛНОМОЧИЯМИ, СОСТОЯВШИЙ ПОД СЛЕДСТВИЕМ ТРЕХ « МАЙОРСКИХ» КАНЦЕЛЯРИЙ, Я.Ф.ДОЛГОРУКОВ НЕ ПОНЕС НИКАКОЙ ОТВЕТСТВЕННОСТИ. БОЛЕЕ ТОГО: ОКАЗАВШИСЬ, ПО ВЫСОЧАЙШЕЙ ВОЛЕ, ПОДСЛЕДСТВЕННЫМ (А ЗАТЕМ И ПОДСУДИМЫМ), КНЯЗЬ ЯКОВ ФЕДОРОВИЧ НИКАК НЕ ПОСТРАДАЛ КАРЬЕРНО. ЦАРЬ НЕ ВЫВЕЛ КНЯЗЯ ИЗ ЧИСЛА СЕНАТОРОВ, СОХРАНИЛ ЗА НИМ ПОСТ ПРЕЗИДЕНТА НОВОУЧРЕЖДЕННОЙ ПО ОБРАЗЦУ ШВЕДСКОЙ Kammarrevisionen РЕВИЗИОН-КОЛЛЕГИИ (НА КАКОВОЙ Я.Ф. ДОЛГОРУКОВ БЫЛ ОПРЕДЕЛЕН, ЧТО ХАРАКТЕРНО, МЕНЕЕ НЕДЕЛИ СПУСТЯ ПОСЛЕ ТОГО, КАК ПОПАЛ ПОД СЛЕДСТВИЕ - 15 ДЕКАБРЯ $\left.1717 \Gamma^{37}\right)$. ВПРОЧЕМ, ВНИМАНИЕ ЦАРЯ ПРИВЛЕКАЛИ НЕ ТОЛЬКО УГОЛОВНЫЕ ДЕЛА С УЧАСТИЕМ САНОВНЫХ ПОДСЛЕДСТВЕННЫХ И ПОДСУДИМЫХ. ВПОЛНЕ АКТИВНОЕ УЧАСТИЕ ПЕТР І ПРИНЯЛ В ПРОЦЕССЕ ПО ОБВИНЕНИЮ ГРУППЫ ОРДИНАРНЫХ СЛУЖАЩИХ РЕВЕЛЬСКОЙ АДМИРАЛТЕЙСКОЙ ПРОВИАНТСКОЙ КАНЦЕЛЯРИИ ВО ВЗЯТКАХ И ХИЩЕНИИ КАЗНЫ (« РЕВЕЛЬСКОМ АДМИРАЛТЕЙСКОМ ДЕЛЕ») ${ }^{38}$. ПОЛУЧИВ СВЕДЕНИЯ О ЗЛОУПОТРЕБЛЕНИЯХ РЕВЕЛЬСКИХ ВОЕННО-МОРСКИХ ИНТЕНДАНТОВ, ЦАРЬ СЧЕЛ ДЕЛО НАСТОЛЬКО ВАЖНЫМ, ЧТО 8 АВГУСТА 1718 Г. РАСПОРЯДИЛСЯ НАПРАВИТЬ ЕГО ДЛЯ ПРЕДВАРИТЕЛЬНОГО РАССЛЕДОВАНИЯ В ТАЙНУЮ КАНЦЕЛЯРИЮ ${ }^{39}$. НЕКОТОРЫЕ ФИГУРАНТЫ ЭТОГО ДЕЛА БЫЛИ В 1720 Г. ОСУЖДЕНЫ ВОЕННЫМ СУДОМ, А КОМИССАР Я. ЛОПУХИН 4 АВГУСТА 1720 Г. ПРАВИТЕЛЬСТВУЮЩИМ СЕНАТОМ ${ }^{40}$.

СТОИТ ЗАМЕТИТЬ, ЧТО ЛИЧНОЕ ПРИСУТСТВИЕ МОНАРХА НА ДОПРОСАХ НЕРЕДКО ОКАЗЫВАЛО НА ПОДСЛЕДСТВЕННЫХ И ПОДСУДИМЫХ ЗНАЧИТЕЛЬНОЕ ПСИХОЛОГИЧЕСКОЕ ВОЗДЕЙСТВИЕ, СПОСОБСТВУЯ ДАЧЕ ПРИЗНАТЕЛЬНЫХ И ОТКРОВЕННЫХ ПОКАЗАНИЙ. К ПРИМЕРУ, ИМЕННО В ХОДЕ ДОПРОСА 5 ФЕВРАЛЯ 1723 Г., ПРОХОДИВШЕГО С УЧАСТИЕМ ИМПЕРАТОРА, СУДЬЯ МОСКОВСКОГО НАДВОРНОГО СУДА БЫВШИЙ ОБЕР-ФИСКАЛ М.В.ЖЕЛЯБУЖСКИЙ ПРИЗНАЛСЯ В ПОДЛОГЕ ЗАВЕЩАНИЯ ВДОВЫ АКУЛИНЫ ПОЛИВАНОВОЙ ${ }^{41}$. 
ИЗ ЧИСЛА СУДЕБНЫХ ОРГАНОВ, ПОВЫШЕННЫЙ ИНТЕРЕС ПЕТРА І ПРИВЛЕКАЛА, КОНЕЧНО, РАБОТА СПЕЦИАЛИЗИРОВАННЫХ СУДОВ ПО ГОСУДАРСТВЕННЫМ ПРЕСТУПЛЕНИЯМ ТАЙНОЙ КАНЦЕЛЯРИИ И ПРЕОБРАЖЕНСКОГО ПРИКАЗА. ПОКАЗАТЕЛЬНО, ЧТО 25 НОЯБРЯ 1718 Г. ЦАРЬ УСТАНОВИЛ СЕБЕ ОСОБЫЙ ДЕНЬ (ПОНЕДЕЛЬНИК) ДЛЯ ПОСЕЩЕНИЯ ТАЙНОЙ КАНЦЕЛЯРИИ ${ }^{42}$.

НАПРОТИВ, С СУДЕБНОЙ ДЕЯТЕЛЬНОСТЬЮ ПРАВИТЕЛЬСТВУЮЩЕГО СЕНАТА МОНАРХ СОПРИКАСАЛСЯ ЛИШЬ ЭПИЗОДИЧЕСКИ. КАК ЯВСТВУЕТ ИЗ АРХИВНЫХ МАТЕРИАЛОВ И ФУНДАМЕНТАЛЬНОЙ ПУБЛИКАЦИИ Н.А. ВОСКРЕСЕНСКОГО, ЗА 1719-1724 ГГ. ПЕТР I НАПРАВИЛ НА СУДЕБНОЕ РАССМОТРЕНИЕ СЕНАТА ЛИШЬ 14 ДЕЛ. НАПРИМЕР, 11 МАРТА 1721 Г. ЦАРЬ ПЕРЕДАЛ В СУДЕБНОЕ ПРОИЗВОДСТВО СЕНАТА РАССЛЕДОВАННОЕ КАНЦЕЛЯРИЕЙ И.И. ДМИТРИЕВА-МАМОНОВА ДЕЛО БЫВШЕГО СИБИРСКОГО ГУБЕРНАТОРА М.П. ГАГАРИНА, А 18 МАЯ 1722 Г. - РАССЛЕДОВАННОЕ КАНЦЕЛЯРИЕЙ С.А.САЛТЫКОВА ДЕЛО БЫВШЕГО ВОРОНЕЖСКОГО ВИЦЕ-ГУБЕРНАТОРА С.А. КОЛЫЧЕВА ${ }^{43}$. ОТНОСЯЩИХСЯ ЖЕ К 1719-1725 ГГ. ФАКТОВ НАПРАВЛЕНИЯ ПЕТРОМ I НА РАССМОТРЕНИЕ СЕНАТА ДЕЛ ИЗ ЮСТИЦ-КОЛЛЕГИИ ВЫЯВИТЬ К НАСТОЯЩЕМУ ВРЕМЕНИ НЕ УДАЛОСЬ. ТАКИМ ОБРАЗОМ, В ЭТОТ ПЕРИОД НОРМЫ, ЗАКРЕПЛЕННЫЕ В СТ. 4 ИМЕННОГО УКАЗА ОТ 19 ДЕКАБРЯ 1718 Г. И В СТ. 4 « ДОЛЖНОСТИ СЕНАТА» ОТ ДЕКАБРЯ 1718 Г., ОСТАЛИСЬ МЕРТВОЙ БУКВОЙ.

В ПОСЛЕДНИЕ ГОДЫ ЖИЗНИ НАИБОЛЬШЕЕ ВНИМАНИЕ ПЕРВЫЙ РОССИЙСКИЙ ИМПЕРАТОР УДЕЛЯЛ УЧРЕЖДЕННОМУ В 1723 Г. В КАЧЕСТВЕ ВЫСШЕГО ОРГАНА ПРАВОСУДИЯ ВЫШНЕМУ СУДУ. В ЧАСТНОСТИ, СОХРАНИЛИСЬ РЕЕСТРЫ ДЕЛ СУДА С МНОГОЧИСЛЕННЫМИ ВЫСОЧАЙШИМИ РЕЗОЛЮЦИЯМИ, НАЛОЖЕННЫМИ 24 ОКТЯБРЯ И 9 ДЕКАБРЯ $1723 \Gamma^{44}$. А В СОСТАВЛЕННОМ ИМПЕРАТОРОМ 13 ЯНВАРЯ 1724 Г. РАСПРЕДЕЛЕНИИ ЗАНЯТИЙ ПО ДНЯМ НЕДЕЛИ ЗНАЧИЛОСЬ : «...В [ВЫШНЕМ] СУДЕ - СЕРЕДЫ ВЕЧЕР И ЧЕТВЕРТКА УТРО» ${ }^{45}$. ДАННЫЙ РАСПОРЯДОК ОТНЮДЬ НЕ ОСТАЛСЯ НА БУМАГЕ : КАК ЯВСТВУЕТ ИЗ ПОХОДНОГО ЖУРНАЛА ПЕТРА І, ЗА ВТОРУЮ ПОЛОВИНУ ЯНВАРЯ 1724 Г. ОН ПОСЕТИЛ ВЫШНИЙ СУД BOCEMЬ $\mathrm{PA}^{46}$.

СИСТЕМАТИЧЕСКИЕ ДАННЫЕ О КОЛИЧЕСТВЕ КАК ВЫНЕСЕННЫХ, ТАК И УТВЕРЖДЕННЫХ ПЕТРОМ I ПРИГОВОРОВ НА СЕГОДНЯ НЕ УСТАНОВЛЕНЫ. ИСХОДЯ ИЗ АРХИВНЫХ МАТЕРИАЛОВ И СВЕДЕНИЙ, ПРИВЕДЕННЫХ В ЛИТЕРАТУРЕ, ВОЗМОЖНО ЛИШЬ ПРЕДПОЛОЖИТЬ, ЧТО КОЛИЧЕСТВО ТАКИХ ПРИГОВОРОВ ИСЧИСЛЯЛОСЬ СОТНЯМИ. НАПРИМЕР, ПО ПОДСЧЕТАМ Н.Б.ГОЛИКОВОЙ, ТОЛЬКО ЗА 1700-1705 ГГ. ПО ДЕЛАМ, НАХОДИВШИМСЯ В ПРОИЗВОДСТВЕ ПРЕОБРАЖЕНСКОГО ПРИКАЗА, ЦАРЬ ВЫНЕС БОЛЕЕ 50 ПРИГОВОРОВ ${ }^{47}$.

В КАЧЕСТВЕ СУДЬИ ПЕТР І ДЕЙСТВОВАЛ НЕ ВСЕГДА СТРОГО ПОСЛЕДОВАТЕЛЬНО. СКАЖЕМ, В 1698 Г. БУДУЩИЙ ИМПЕРАТОР ПРИГОВОРИЛ К СМЕРТНОЙ КАЗНИ ЖЕНУ СТРЯПЧЕГО КОНЮХА АКСИНЬЮ ТРУСОВУ И ЕЕ ХОЛОПА ГРИГОРИЯ ЛЕОНТЬЕВА. ОСУЖДЕННЫЕ БЫЛИ ИЗОБЛИЧЕНЫ ПРЕОБРАЖЕНСКИМ ПРИКАЗОМ В ПРОИЗНЕСЕНИИ « НЕПРИСТОЙНЫХ РЕЧЕЙ». БЕСЕДУЯ МЕЖДУ СОБОЙ О ЖЕСТОКОСТИ ЦАРСКОЙ РАСПРАВЫ НАД ВОССТАВШИМИ СТРЕЛЬЦАМИ, ОНИ ПРИШЛИ К КРАМОЛЬНОМУ ВЫВОДУ О ТОМ, ЧТО ПЕТР І « ОЖИДОВЕЛ И БЕС ТОВО ДЕ ОН ЖИТЬ НЕ МОЖЕТ, ЧТОБ ЕМУ НЕКОТОРЫЙ ДЕНЬ КРОВИ НЕ ПИТЬ». МЕЖДУ ТЕМ, ТРИ ГОДА СПУСТЯ, В ЯВСТВЕННО СХОДНОМ СЛУЧАЕ, ЦАРЬ ВЫНЕС БОЛЕЕ МЯГКИЙ ПРИГОВОР. В 1701 Г. ОН ОСУДИЛ К НАКАЗАНИЮ КНУТОМ, УРЕЗАНИЮ ЯЗЫКА И ПОЖИЗНЕННОЙ ССЫЛКЕ В МОНАСТЫРЬ В ВОЛОГДЕ ПОСАДСКУЮ ДОЧЬ ЕВДОКИЮ ЧАСОВНИКОВУ, ПРИЗНАННУЮ ВИНОВНОЙ В ПРОИЗНЕСЕНИИ ФРАЗЫ : « КОТОРОГО ДЕ ДНИ ВЕЛИКИЙ ГОСУДАРЬ И СТОЛЬНИК КНЯЗЬ РОМОДАНОВСКИЙ КРОВИ ИЗОПЬЮТ, ТОГО ДЕ ДНИ 
В ТЕ ЧАСЫ ОНИ ВЕСЕЛЫ, А КОТОРОГО ДНИ ОНИ КРОВИ НЕ ИЗОПЬЮТ, И ТОГО ДНИ ИМ И ХЛЕБ НЕ ЕС[Т]ЦА» ${ }^{48}$. НОЯБРЯ 1718 Г. ОСОБЫМ ВОЕННО-СУДЕБНЫМ ПРИСУТСТВИЕМ К СМЕРТНОЙ КАЗНИ. ВМЕСТО КАЗНИ ЦАРЬ ОПРЕДЕЛИЛ ИВАНУ ТАРБЕЕВУ ВЫРЕЗАНИЕ НОЗДРЕЙ И ПОЖИЗНЕННУЮ ССЫЛКУ НА КАТОРГУ 55 . СХОДНЫМ ОБРАЗОМ БУДУЩИЙ ИМПЕРАТОР ПОСТУПИЛ И С ПРИГОВОРЕННЫМ В 1720 Г. СЕНАТОМ К СМЕРТНОЙ КАЗНИ ФИГУРАНТОМ 
« РЕВЕЛЬСКОГО АДМИРАЛТЕЙСКОГО ДЕЛА» ЯКОВОМ ЛОПУХИНЫМ. ВЗАМЕН КАЗНИ ЕМУ БЫЛИ НАЗНАЧЕНЫ НАКАЗАНИЕ КНУТОМ, ВЫРЕЗАНИЕ НОЗДРЕЙ И ВЕЧНАЯ КАТОРГА ${ }^{56}$.

А ВОТ НАЧАЛЬНИКУ МОРСКОГО КОМИССАРИАТА ГЕНЕРАЛ-МАЙОРУ Г.П. ЧЕРНЫШЕВУ, ОСУЖДЕННОМУ 6 ОКТЯБРЯ 1718 Г. ОСОБЫМ ВОЕННО-СУДЕБНЫМ ПРИСУТСТВИЕМ ЗА ЗЛОУПОТРЕБЛЕНИЯ ПРИ СТРОИТЕЛЬСТВЕ САНКТ-ПЕТЕРБУРГСКОГО ДОМА К ЛИШЕНИЮ ЧИНОВ И КОНФИСКАЦИИ ИМУЩЕСТВА, ПЕТР І ПРИ УТВЕРЖДЕНИИ ПРИГОВОРА СНИЗИЛ НАКАЗАНИЕ ДО АРЕСТА НА ПЯТЬ ДНЕЙ И ШТРАФА В 372 РУБЛЯ ${ }^{57}$. СМЯГЧИЛ ИМПЕРАТОР И ПРИГОВОР, ВЫНЕСЕННЫЙ ОСОБЫМ СУДЕБНЫМ ПРИСУТСТВИЕМ СЕНАТОРУ П.П. ШАФИРОВУ : В КАЧЕСТВЕ ЗАМЕНЫ СМЕРТНОЙ КАЗНИ ОСУЖДЕННОМУ БЫЛИ ОПРЕДЕЛЕНЫ ССЫЛКА В ЯКУТИЮ, ЛИШЕНИЕ ЧИНОВ И КОНФИСКАЦИЯ ИМУЩЕСТВА ${ }^{58}$. ПРИЗНАННОМУ ВЫШНИМ СУДОМ ВИНОВНЫМ В ПОЛУЧЕНИИ ДВУХ ВЗЯТОК И ХАЛАТНОСТИ КРОНШТАДТСКОМУ ТАМОЖЕННОМУ БУРМИСТРУ Ф.А.БОЛОТНОМУ ПЕТР І СМЯГЧИЛ ПРИГОВОР И ВОВСЕ ДВАЖДЫ. ДЛЯ НАЧАЛА, ПРИСУТСТВУЯ НА ЗАСЕДАНИИ ВЫШНЕГО СУДА 15 ЯНВАРЯ 1724 Г., ИМПЕРАТОР ОТМЕНИЛ РЕШЕНИЕ СУДА О СМЕРТНОЙ КАЗНИ БЫВШЕГО БУРМИСТРА, НАЗНАЧИВ ЕМУ ВЗАМЕН НАКАЗАНИЕ КНУТОМ И ССЫЛКУ НА КАТОРГУ НА ПЯТЬ ЛЕТ. ЗАТЕМ, УТВЕРЖДАЯ ПРИГОВОР, ВЫНЕСЕННЫЙ ВЫШНИМ СУДОМ 23 ЯНВАРЯ 1724 Г., ПЕТР І СОКРАТИЛ СРОК НАЗНАЧЕННОЙ ФЕДОРУ БОЛОТНОМУ КАТОРГИ ДО ТРЕХ ЛЕТ 59 .

ЗАМЕТНО СМЯГЧИЛ ГЛАВА ГОСУДАРСТВА И ПРИГОВОР УЛИЧЕННОМУ В ПОСОБНИЧЕСТВЕ ОБЕР-ФИСКАЛУ А.Я. НЕСТЕРОВУ ПОДЬЯЧЕМУ Ф. ЩЕТИНИНУ, КОТОРОГО ВЫШНИЙ СУД ПРИГОВОРИЛ 23 ЯНВАРЯ 1724 Г. К НАКАЗАНИЮ КНУТОМ, ВЫРЕЗАНИЮ НОЗДРЕЙ И ПОЖИЗНЕННОЙ КАТОРГЕ. НА ДАННЫЙ ПРИГОВОР ПЕТР І НАЛОЖИЛ УТВЕРЖДАЮЩУЮ РЕЗОЛЮЦИЮ : « КРОМЕ ВЫРЕЗАНИЯ НОЗДРЕЙ, А ВРЕМЯ НА 10 ЛЕТ ${ }^{60}$. ПОМИЛОВАЛ ИМПЕРАТОР ТАКЖЕ БЫВШЕГО ЯРОСЛАВСКОГО ФИСКАЛА А.И. НИКИТИНА, ПРИГОВОРЕННОГО ВЫШНИМ СУДОМ 22 ЯНВАРЯ 1724 Г. ЗА ПОЛУЧЕНИЕ ТРЕХ ВЗЯТОК К СМЕРТНОЙ КАЗНИ. НА ПРИГОВОРЕ АЛЕКСЕЮ НИКИТИНУ ПЕТР І ЛАКОНИЧНО НАЧЕРТАЛ : « ПОЛИТИЧЕСКОЮ СМЕРТИЮ», - ЧТО ПОДРАЗУМЕВАЛО ВЫРЕЗАНИЕ НОЗДРЕЙ, НАКАЗАНИЕ КНУТОМ И ССЫЛКУ НА КАТОРГУ. БОЛЕЕ ТОГО : КАК ЯВСТВУЕТ ИЗ ПОМЕТЫ В СУДНОМ ДЕЛЕ, ПЕРЕД САМЫМ ПРИВЕДЕНИЕМ ПРИГОВОРА В ИСПОЛНЕНИЕ ПАЛАЧ ПОЛУЧИЛ УКАЗАНИЕ НЕ НАНОСИТЬ А.И. НИКИТИНУ БОЛЕЕ 25 УДАРОВ КНУТОМ, ЧТО ЗНАЧИТЕЛЬНО ПОВЫШАЛО ШАНСЫ ОСУЖДЕННОГО ВЫЖИТЬ ПОСЛЕ ТЕЛЕСНОГО НАКАЗАНИЯ ${ }^{61}$. ИЗ ШЕСТИ ВЫСШИХ ДОЛЖНОСТНЫХ ЛИЦ, ПРИГОВОРЕННЫХ В ПЕРВОЙ ЧЕТВЕРТИ XVIII В. РАЗЛИЧНЫМИ СУДЕБНЫМИ ОРГАНАМИ К СМЕРТНОЙ КАЗНИ ЗА ПРЕСТУПЛЕНИЯ ПРОТИВ ИНТЕРЕСОВ СЛУЖБЫ, КАЗНЕНО БЫЛО ЛИШЬ ДВОЕ. ПОДТВЕРЖДАЮЩИЕ РЕЗОЛЮЦИИ (« УЧИНИТЬ ПО СЕНАТСКОМУ ПРИГОВОРУ» И « БЫТЬ ПО СЕМУ») ПЕТР І НАЛОЖИЛ ЛИШЬ НА СМЕРТНЫЕ ПРИГОВОРЫ, ВЫНЕСЕННЫЕ ПРАВИТЕЛЬСТВУЮЩИМ СЕНАТОМ 14 МАРТА 1721 Г. БЫВШЕМУ СИБИРСКОМУ ГУБЕРНАТОРУ М.П.ГАГАРИНУ И ВЫШНИМ СУДОМ 22 ЯНВАРЯ 1724 Г. БЫВШЕМУ ГЛАВЕ ФИСКАЛЬСКОЙ СЛУЖБЫ А.Я. НЕСТЕРОВУ ${ }^{62}$. ПРИЧИНЫ, ПО КОТОРЫМ ПЕТР І СМЯГЧАЛ ПРИГОВОРЫ, ДАЛЕКО НЕ ВСЕГДА ПОНЯТНЫ, ТЕМ БОЛЕЕ ЧТО САМ ОН РАЗЪЯСНЯЛ ИХ КРАЙНЕ РЕДКО. ИСХОДЯ ИЗ ОСОБЕННОСТЕЙ НАТУРЫ ПЕРВОГО РОССИЙСКОГО ИМПЕРАТОРА, ОТЛИЧАВШЕГОСЯ ОЧЕВИДНЫМ БЕЗРАЗЛИЧИЕМ К ЛЮДСКИМ СТРАДАНИЯМ, ВОЗМОЖНО С УВЕРЕННОСТЬЮ ПРЕДПОЛОЖИТЬ, ЧТО, ОТМЕНЯЯ СМЕРТНЫЕ ПРИГОВОРЫ, ОН РУКОВОДСТВОВАЛСЯ ОТНЮДЬ НЕ СООБРАЖЕНИЯМИ ГУМАННОСТИ. РЕЛИГИОЗНО ОКРАШЕННЫЙ ТРАДИЦИОННЫЙ ОБРАЗ МИЛОСЕРДНОГО 
ГОСУДАРЯ», СУДЯ ПО ВСЕМУ, НИЧУТЬ НЕ ПРИВЛЕКАЛ ПЕТРА І, И ОН ВОВСЕ НЕ СТРЕМИЛСЯ СОЗДАТЬ ТАКОЕ ПРЕДСТАВЛЕНИЕ О СЕБЕ У ПОДДАННЫХ.

КАК ПРЕДСТАВЛЯЕТСЯ, ПРАГМАТИЧНО НАСТРОЕННЫЙ И НЕ ЛИШЕННЫЙ ПРЕДСТАВЛЕНИЙ О СПРАВЕДЛИВОСТИ, ПЕТР I СТАРАЛСЯ РЕШАТЬ УЧАСТЬ ОСУЖДЕННЫХ ДИФФЕРЕНЦИРОВАННО, ИСХОДЯ, С ОДНОЙ СТОРОНЫ, ИЗ ПРЕДСТАВЛЕНИЙ О СТЕПЕНИ ОБЩЕСТВЕННОЙ ОПАСНОСТИ ИНКРИМИНИРОВАННЫХ ИМ ДЕЯНИЙ, А С ДРУГОЙ - ИЗ УЧЕТА ИХ ЗАСЛУГ ПЕРЕД ГОСУДАРСТВОМ. К ТОМУ ЖЕ ПЕРВЫЙ РОССИЙСКИЙ ИМПЕРАТОР ВПОЛНЕ ТРЕЗВО ОСОЗНАВАЛ ИЗБИРАТЕЛЬНОСТЬ УГОЛОВНОЙ РЕПРЕССИИ ПО ДЕЛАМ О ДОЛЖНОСТНЫХ ПРЕСТУПЛЕНИЯХ. ПОЭТОМУ, К ПРИМЕРУ, ЕСЛИ ПОЛУЧЕНИЕ ГОСУДАРСТВЕННЫМ СЛУЖАЩИМ НЕЗАКОННОГО ВОЗНАГРАЖДЕНИЯ НЕ ПРИВЕЛО К СОВЕРШЕНИЮ ИНОГО СЕРЬЕЗНОГО ПРЕСТУПЛЕНИЯ ПРОТИВ ИНТЕРЕСОВ СЛУЖБЫ, ПЕТР І ПРЕДПОЧИТАЛ ПОСТУПАТЬ С ВИНОВНЫМ В БОЛЬШЕЙ МЕРЕ СНИСХОДИТЕЛЬНО. ИМЕННО ПОЭТОМУ, ДУМАЕТСЯ, В ЯНВАРЕ 1724 Г. БЫЛИ ПОМИЛОВАНЫ ОРДИНАРНЫЕ ВЗЯТОЧНИКИ А.И. НИКИТИН И Ф.А. БОЛОТНЫЙ.

В СЛУЧАЕ ЖЕ С ГЕНЕРАЛ-МАЙОРОМ Г.П. ЧЕРНЫШЕВЫМ, ИЗОБЛИЧЕННЫМ В НЕЗАКОННОЙ ПОКУПКЕ КАЗЕННОГО ЛЕСА ДЛЯ ПОСТРОЙКИ ДОМА В САНКТ-ПЕТЕРБУРГЕ И В ИСПОЛЬЗОВАНИИ НА СТРОИТЕЛЬСТВЕ ДОМА СОЛДАТ И КАТОРЖАН, ПЕТР І, НЕСОМНЕННО, ПРИНЯЛ ВО ВНИМАНИЕ НЕ ТОЛЬКО СРАВНИТЕЛЬНО НЕЗНАЧИТЕЛЬНУЮ ОПАСНОСТЬ ПРЕСТУПНОГО ДЕЯНИЯ, НО И БОЕВОЕ ПРОШЛОЕ ОСУЖДЕННОГО. ГРИГОРИЙ ЧЕРНЫШЕВ ЯВЛЯЛСЯ ЗАСЛУЖЕННЫМ ФРОНТОВИКОМ, ПОЛУЧИВШИМ ПЯТЬ РАНЕНИЙ В БОЯХ ВЕЛИКОЙ СЕВЕРНОЙ ВОЙНЫ (ЧТО, СТОИТ НАПОМНИТЬ, ОТНЮДЬ НЕ ПОЛНОСТЬЮ ОСВОБОДИЛО ЕГО ОТ ОТВЕТСТВЕННОСТИ $\left.{ }^{63}\right)$. БЛАГОДАРЯ СВОИМ ДИПЛОМАТИЧЕСКИМ ЗАСЛУГАМ, В 1723 Г. СОХРАНИЛ ЖИЗНЬ П.П. ШАФИРОВ, ОСУЖДЕННЫЙ ЗА СЛУЖЕБНЫЙ ПОДЛОГ И ЗЛОУПОТРЕБЛЕНИЕ ДОЛЖНОСТНЫМИ ПОЛНОМОЧИЯМИ. В УКАЗЕ, ЗАЧИТАННОМ 15 ФЕВРАЛЯ 1723 Г. ПЕТРУ ШАФИРОВУ НА ЭШАФОТЕ, ПРЯМО ГОВОРИЛОСЬ О ТОМ, ЧТО ИМПЕРАТОР ПРИНЯЛ РЕШЕНИЕ ПОМИЛОВАТЬ ЕГО, «НАПОМИНАЯ ПРЕЖНИЕ ТВОИ слУжБЫ» ${ }^{64}$.

В СВОЮ ОЧЕРЕДЬ, ХОЛОПУ АЛЕКСЕЮ НЕМИРОВУ, ОСУЖДЕННОМУ В 1700 Г. ЗА ЛОЖНОЕ ОБВИНЕНИЕ ХОЗЯИНА В ГОСУДАРСТВЕННОМ ПРЕСТУПЛЕНИИ, ПЕТР І ЗАМЕНИЛ СМЕРТНУЮ КАЗНЬ НА НАКАЗАНИЕ КНУТОМ, КЛЕЙМЕНИЕ И ТРЕХЛЕТНИЕ КАТОРЖНЫЕ РАБОТЫ, ПРИНЯВ ВО ВНИМАНИЕ ЕГО НЕСОВЕРШЕННОЛЕТИЕ («ЧТО ОН, АЛЕШКА, НЕ В СОВЕРШЕННЫХ ЛЕТЕХ» $)^{65}$. ЕЩЕ БОЛЕЕ ПРИМЕЧАТЕЛЕН БЫЛ ХОД МЫСЛИ ИМПЕРАТОРА ПРИ РАЗРЕШЕНИИ ВОПРОСА О СУДЬБЕ БЫВШЕГО ХОЛОПА Ф. РЕЗАНОВА, ПРИГОВОРЕННОГО ВЫШНИМ СУДОМ 23 ЯНВАРЯ 1724 Г. К СМЕРТНОЙ КАЗНИ ЗА ПОСОБНИЧЕСТВО А.Я. НЕСТЕРОВУ В ВЫМОГАТЕЛЬСТВЕ ВЗЯТОК С ЖИТЕЛЕЙ РЖЕВА. В РЕЗОЛЮЦИИ НА ПРИГОВОРЕ ПЕТР І ОТМЕТИЛ, ЧТО ФЕДОР РЕЗАНОВ НЕ ЯВЛЯЛСЯ В МОМЕНТ СОВЕРШЕНИЯ ПРЕСТУПЛЕНИЯ ДОЛЖНОСТНЫМ ЛИЦОМ (« НИКАКОГО ДЕЛА ВРУЧЕННОГО НЕ ИМЕЛ»), НА ОСНОВАНИИ ЧЕГО ЗАМЕНИЛ ЕМУ КАЗНЬ НА ПОЛИТИЧЕСКУЮ СМЕРТЬ» (ЧТО, КАК И В СЛУЧАЕ С А.И. НИКИТИНЫМ, ОЗНАЧАЛО НАКАЗАНИЕ КНУТОМ, ВЫРЕЗАНИЕ НОЗДРЕЙ И ССЫЛКУ НА КАТОРГУ) ${ }^{66}$.

ДОСТОЕН ОТДЕЛЬНОГО УПОМИНАНИЯ ЭПИЗОД, КОГДА РОССИЙСКИЙ МОНАРХ ВПЕРВЫЕ ОСОЗНАЛ НЕДОПУСТИМОСТЬ ПРИДАНИЯ УГОЛОВНОМУ ЗАКОНУ ОБРАТНОЙ СИЛЫ. ЭТО ПРОИЗОШЛО В 1722 Г., КОГДА К ПЕТРУ І ПОСТУПИЛ НА УТВЕРЖДЕНИЕ ПРИГОВОР ВОЕННОГО СУДА, ВЫНЕСЕННЫЙ НА ОСНОВАНИИ АРТИКУЛА ВОИНСКОГО 1714 Г. БЫВШЕМУ ОБЕР-КОМЕНДАНТУ АСТРАХАНИ ГВАРДИИ ПОРУЧИКУ М.И. ЧИРИКОВУ, ИЗОБЛИЧЕННОМУ В 
МНОГОЧИСЛЕННЫХ ПРЕСТУПЛЕНИЯХ ПРОТИВ ИНТЕРЕСОВ СЛУЖБЫ. НЕСМОТРЯ НА ТО, ЧТО МИХАИЛ ЧИРИКОВ ОБВИНЯЛСЯ В ПРЕСТУПЛЕНИЯХ, СОВЕРШЕННЫХ В 1717-1718 ГГ., ИМПЕРАТОР, ПРОЯВИВ НЕОРДИНАРНУЮ ЮРИДИЧЕСКУЮ ЩЕПЕТИЛЬНОСТЬ, ПРЕДПИСАЛ ВЫЯСНИТЬ, ИМЕЛ ЛИ БЫВШИЙ ОБЕР-КОМЕНДАНТ ВОЗМОЖНОСТЬ ОЗНАКОМИТЬСЯ С АРТИКУЛОМ ВОИНСКИМ («БЫЛ ЛИ В ПРИСЫЛКЕ В АСТРАХАНЬ... АРТИКУЛ ВОЕННЫЙ»). ПОСКОЛЬКУ, КАК БЫЛО УСТАНОВЛЕНО ПУТЕМ ОПРОСА ПОДЬЯЧИХ КОМЕНДАНТСКОЙ КАНЦЕЛЯРИИ И ОФИЦЕРОВ МЕСТНОГО ГАРНИЗОНА, НИ ОДНОГО ЭКЗЕМПЛЯРА АРТИКУЛА ВОИНСКОГО В АСТРАХАНЬ В ПЕРИОД ПРЕБЫВАНИЯ ТАМ М.И. ЧИРИКОВА НЕ ПОСТУПАЛО, ПЕТР I НЕ СТАЛ УТВЕРЖДАТЬ ПРИГОВОР, УКАЗАВ ПОДГОТОВИТЬ ЕГО НОВЫЙ ВАРИАНТ (ЧТО ОЗНАЧАЛО НЕОБХОДИМОСТЬ ПЕРЕКВАЛИФИЦИРОВАТЬ ДОКАЗАННЫЕ СУДОМ ЭПИЗОДЫ ПРЕСТУПНОЙ ДЕЯТЕЛЬНОСТИ БЫВШЕГО ОБЕР-КОМЕНДАНТА НА ОСНОВАНИИ ИНЫХ АКТОВ УГОЛОВНОГО ЗАКОНОДАТЕЛЬСТВА) $)^{67}$.

51 НАИБОЛЕЕ ЖЕ ЯРКИМ ПРИМЕРОМ ПРИВЕРЖЕННОСТИ ПЕРВОГО РОССИЙСКОГО ИМПЕРАТОРА « РЕГУЛЯРНОМУ» СУДОПРОИЗВОДСТВУ ПРЕДСТАВЛЯЕТСЯ ПРОВЕДЕННЫЙ В НОЯБРЕ 1724 Г. ПРОЦЕСС НАД КАМЕРГЕРОМ ГВАРДИИ ПОРУЧИКОМ В. МОНСОМ. ПРИ ВСЕМ ТОМ, ЧТО ВИЛИМ МОНС БЫЛ УЛИЧЕН В РАЗНООБРАЗНЫХ ПРЕСТУПНЫХ ДЕЯНИЯХ (ГЛАВНЫМ ОБРАЗОМ, В ПОЛУЧЕНИИ ВЗЯТОК И ПРЕВЫШЕНИИ ДОЛЖНОСТНЫХ ПОЛНОМОЧИЙ), В ДЕЛЕ ИМЕЛСЯ ЕЩЕ ОДИН АСПЕКТ : ОТКРЫЛОСЬ, ЧТО КАМЕРГЕР СОСТОЯЛ В НЕПОДОБАЮЩИХ ОТНОШЕНИЯХ С ИМПЕРАТРИЦЕЙ ЕКАТЕРИНОЙ АЛЕКСЕЕВНОЙ ${ }^{68}$. В ЭТИХ УСЛОВИЯХ, ИМЕЯ ПОЛНУЮ ВОЗМОЖНОСТЬ ОСУДИТЬ В. МОНСА ЕДИНОЛИЧНО ИЛИ ВОВСЕ РАСПРАВИТЬСЯ С НИМ ВО ВНЕСУДЕБНОМ ПОРЯДКЕ, ПЕТР І ПРЕДПОЧЕЛ СОБЛЮСТИ ВСЕ ПРОЦЕДУРЫ ОРДИНАРНОГО СУДЕБНОГО ПРОИЗВОДСТВА. В ИТОГЕ, ПОСЛЕ ПРЕДВАРИТЕЛЬНОГО СЛЕДСТВИЯ (ОСУЩЕСТВЛЕННОГО В ВЕСЬМА СЖАТЫЕ СРОКИ ИМПЕРАТОРСКИМ КАБИНЕТОМ) ДЕЛО В. МОНСА БЫЛО НАПРАВЛЕНО В ВЫШНИЙ СУД, КОТОРЫЙ ВЫНЕС ЕМУ 14 НОЯБРЯ 1723 Г. СМЕРТНЫЙ ПРИГОВОР, НЕЗАМЕДЛИТЕЛЬНО УТВЕРЖДЕННЫЙ МОНАРХОМ ${ }^{69}$.

ОСТАЕТСЯ ДОБАВИТЬ, ЧТО ПРИ ВЫНЕСЕНИИ И УТВЕРЖДЕНИИ ПРИГОВОРОВ - В КАЧЕСТВЕ ДОПОЛНИТЕЛЬНОЙ САНКЦИИ - ПЕТР І РЕГУЛЯРНО ПРАКТИКОВАЛ ТАКУЮ СВОЕОБРАЗНУЮ МЕРУ КАК ЗАПРЕТ ПОГРЕБАТЬ ТЕЛА ЛИЦ, КАЗНЕННЫХ ЗА ГОСУДАРСТВЕННЫЕ ПРЕСТУПЛЕНИЯ И ЗА ПРЕСТУПЛЕНИЯ ПРОТИВ ИНТЕРЕСОВ СЛУЖБЫ ${ }^{70}$. ТАК, ПРИГОВОРИВ 24 ФЕВРАЛЯ 1712 Г. К СМЕРТНОЙ КАЗНИ ИЗОБЛИЧЕННОГО МОСКОВСКОЙ ГУБЕРНСКОЙ КАНЦЕЛЯРИЕЙ В НЕУКАЗНЫХ СБОРАХ И ВЗЯТОЧНИЧЕСТВЕ КОМЕНДАНТА Г. ЛУХА Ф.А. ВОЛКОВА, ЦАРЬ СПЕЦИАЛЬНО ПРЕДПИСАЛ ТРУП ЕВО В ЗЕМЛЮ НЕ ХОРОНИТЬ (НО ЧТОБ ЛЕЖАЛ ПОВЕРХ ЗЕМЛИ, ВИДИМ ВСЕМ)» ${ }^{71}$. НЕ БЫЛИ ПРЕДАНЫ ЗЕМЛЕ И ТЕЛА КАЗНЕННЫХ ПО ДЕЛУ ЦАРЕВИЧА АЛЕКСЕЯ ПЕТРОВИЧА - В ЧАСТНОСТИ, ШЕСТЕРЫХ ЛИЦ, ОБЕЗГЛАВЛЕННЫХ В САНКТ-ПЕТЕРБУРГЕ 8 ДЕКАБРЯ $1718 \Gamma^{72}$. ОБЩЕИЗВЕСТНО, ЧТО БЫЛО ОСТАВЛЕНО НА ВИСЕЛИЦЕ ТЕЛО КАЗНЕННОГО 16 МАРТА 1721 Г. М.П. ГАГАРИНА ${ }^{73}$. НЕ БЫЛО ПОГРЕБЕНО И ТЕЛО ОБЕЗГЛАВЛЕННОГО 15 НОЯБРЯ 1724 Г. В. МОНСА ${ }^{74}$.

53 ТЯГОТЕНИЕ ПЕРВОГО РОССИЙСКОГО ИМПЕРАТОРА К ПОДОБНОЙ ФОРМЕ ДОПОЛНИТЕЛЬНОГО УГОЛОВНОГО НАКАЗАНИЯ ПРИВЕЛО К УНИКАЛЬНОМУ СЛУЧАЮ, КОГДА, ПОЛУЧИВ СВЕДЕНИЯ, ПОДТВЕРДИВШИЕ ЭПИЗОДЫ КАЗНОКРАДСТВА УМЕРШЕГО К ТОМУ ВРЕМЕНИ В ХОДЕ ПРЕДВАРИТЕЛЬНОГО СЛЕДСТВИЯ ДЬЯКА П.К. СКУРИХИНА, ПЕТР І ВЫНЕС ЕМУ ПОСМЕРТНЫЙ (!) ПРИГОВОР. 15 АПРЕЛЯ 1724 Г. ИМПЕРАТОР УКАЗАЛ ИЗВЛЕЧЬ ТЕЛО ПЕТРА СКУРИХИНА ИЗ МОГИЛЫ И ПОВЕСИТЬ ЕГО « НА ЖЕЛЕЗНОЙ ЧЕПЕ ЗА МОСКВОЮ РЕКОЮ НА БОЛОТЕ... ЗА ТО, ЧТО ОН, ПОКРЫВАЯ И ОТБЫВАЯ СВОЕГО ВОРОВСТВА, ВО ВСЕМ 
ЗАПИРАЛСЯ И... ПОВИННОЙ НЕ ПРИНЕС» ${ }^{75}$. В ТАКОМ КОНТЕКСТЕ НЕ ПРИХОДИТЬСЯ УДИВЛЯТЬСЯ ОСТОРОЖНОСТИ, ПРОЯВЛЕННОЙ В 1721 Г. ГЛАВОЙ ОДНОЙ ИЗ « МАЙОРСКИХ» СЛЕДСТВЕННЫХ КАНЦЕЛЯРИЙ М.А. МАТЮШКИНЫМ. УЗНАВ О ПОСЛЕДОВАВШЕЙ 29 ИЮНЯ 1721 Г. СМЕРТИ ПОДСЛЕДСТВЕННОГО, БЫВШЕГО АРХАНГЕЛОГОРОДСКОГО ВИЦЕ-ГУБЕРНАТОРА А.А. КУРБАТОВА, МИХАИЛ МАТЮШКИН НЕ РЕШИЛСЯ ДАТЬ РАЗРЕШЕНИЕ НА ЕГО ЗАХОРОНЕНИЕ, А ЗАПРОСИЛ ОСОБОЕ УКАЗАНИЕ ПЕТРА І НА ЭТОТ CYET $^{76}$. ТАК, НЕЛЬЗЯ ИСКЛЮЧИТЬ, ЧТО ОПРЕДЕЛЕНИЕ МОНАРХОМ-РЕФОРМАТОРОМ ТЕХ ИЛИ ИНЫХ ВИДОВ НАКАЗАНИЙ, ОТМЕЧЕННЫЕ ЭПИЗОДЫ НЕПОГРЕБЕНИЯ ТЕЛ КАЗНЕННЫХ ИМЕЛИ И НЕКОЕ МАГИКО-МИФОЛОГИЧЕСКОЕ ЗНАЧЕНИЕ. УСТАНОВЛЕНИЕ ИНЫХ АСПЕКТОВ ДЕЯТЕЛЬНОСТИ ПЕТРА І В ОБЛАСТИ ЮСТИЦИИ ДОЛЖНО БЫТЬ ОСУЩЕСТВЛЕНО В РАМКАХ ДАЛЬНЕЙШИХ ИЗЫСКАНИЙ. КАК ВЕСЬМА АКТИВНЫЙ СУДЕБНЫЙ ДЕЯТЕЛЬ, ХОТЯ И ОДНОСТОРОННЕ СОСРЕДОТОЧЕННЫЙ НА УЧАСТИИ В УГОЛОВНОМ СУДОПРОИЗВОДСТВЕ. В ЦЕНТРЕ ВНИМАНИЯ ПЕРВОГО РОССИЙСКОГО ИМПЕРАТОРА НЕИЗМЕННО НАХОДИЛИСЬ ДЕЛА ПО ГОСУДАРСТВЕННЫМ ПРЕСТУПЛЕНИЯМ И ПО ПРЕСТУПЛЕНИЯМ ПРОТИВ ИНТЕРЕСОВ СЛУЖБЫ.

ПРИ РАССМОТРЕНИИ УГОЛОВНЫХ ДЕЛ ПЕТР І ВЫСТУПАЛ В САМЫХ РАЗНООБРАЗНЫХ ПРОЦЕССУАЛЬНЫХ РОЛЯХ - ОТ ИНИЦИАТОРА УГОЛОВНОГО ПРЕСЛЕДОВАНИЯ ДО ВЕРХОВНОГО СУДЬИ, ВЫНОСИВШЕГО ИЛИ УТВЕРЖДАВШЕГО ПРИГОВОР. УТВЕРЖДАЯ ПРИГОВОРЫ, ПЕРВЫЙ РОССИЙСКИЙ ИМПЕРАТОР ПРЕДПОЧИТАЛ В БОЛЬШЕЙ МЕРЕ 
ПРАГМАТИЧНЫЙ ДИФФЕРЕНЦИРОВАННЫЙ ПОДХОД, НЕ УЖЕСТОЧАЯ, А, НАПРОТИВ, НЕРЕДКО СМЯГЧАЯ НАЗНАЧЕННЫЕ СУДОМ НАКАЗАНИЯ. ПРЕДСТАВЛЯЕТСЯ НЕОСПОРИМЫМ, ЧТО В СВОЕЙ ГОСУДАРСТВЕННОЙ ДЕЯТЕЛЬНОСТИ ПЕТР І В ПОЛНОЙ МЕРЕ ВОПЛОТИЛ В ЖИЗНЬ СЛОВА «ПРАВДЫ ВОЛИ МОНАРШЕЙ» О ТОМ, ЧТО « ДОЛЖЕН ЦАРЬ ПЕЩИСЯ ДА БУДЕТ ИСТИННОЕ В ГОСУДАРСТВЕ ПРАВОСУДИЕ».

\section{NOTES}

1. В ПОСЛЕДНИЕ ДЕСЯТИЛЕТИЯ ТОЧКА ЗРЕНИЯ, ЧТО ФЕОФАН ПРОКОПОВИЧ ЯВИЛСЯ ЕДИНОЛИЧНЫМ АВТОРОМ «ПРАВДЫ ВОЛИ МОНАРШЕЙ», ПОДВЕРГНУТА СОМНЕНИЮ. СМ. : J. Cracraft, « Did Feofan Procopovich Really Write Pravda Voli Monarshei ?», Slavic Review, 40 (2), 1981, с. 173-193 ; э. ЛЕНТИН, « АВТОРСТВО « ПРАВДЫ ВОЛИ МОНАРШЕЙ» : ФЕОФАН ПРОКОПОВИЧ, АФАНАСИЙ КОНДОИДИ», В Н.Д. КОЧЕТКОВА, ОТВ. РЕД., ХVІІІ ВЕК, СБ. 21, СПБ. : НАУКА, 1999, С. $33-$ 39. ПРИ ВСЕЙ УБЕДИТЕЛЬНОСТИ МНОГИХ ДОВОДОВ НАЗВАННЫХ АВТОРОВ, ПРЕДСТАВЛЯЕТСЯ ОЧЕВИДНЫМ, ЧТО ИМЕННО ФЕОФАН СЫГРАЛ КЛЮЧЕВУЮ РОЛЬ В ПОДГОТОВКЕ ЭТОГО СОЧИНЕНИЯ.

2. ПРАВДА ВОЛИ МОНАРШЕЙ ВО ОПРЕДЕЛЕНИИ НАСЛЕДНИКА ДЕРЖАВЫ СВОЕЙ, М.: МОСКОВСКАЯ ТИПОГР., 1722, С. 26.

3. ТАM ЖЕ.

4. C. Schmidt, Sozialkontrolle in Moskau : Justiz, Kriminalität und Leibeigenschaft : 1649-1785, Stuttgart : F. Steiner Verlag, 1996, c. 116-169 ; N.S. Kollmann, Crime and Punishment in Early Modern Russia, Cambridge : Cambridge University Press, 2012, с. 177-202, 258-279. УМЕСтно ОТМЕТИТЬ, чТО В НАЗВАННОЙ МОНОГРАФИИ Н. КОЛЛМАНН ПОДРОБНО РАССМОТРЕЛА ВОПРОС ОБ ОТНОШЕНИИ ПЕТРА І К СМЕРТНОЙ КАЗНИ И ПРОЦЕДУРЕ ЕЕ ИСПОЛНЕНИЯ (С. 403-415).

5. ВОПРОС О ТАКОВОЙ РЕГЛАМЕНТАЦИИ В ДОПЕТРОВСКИЙ И ПОСЛЕПЕТРОВСКИЙ ПЕРИОДЫ ТРЕБУЕТ ДАЛЬНЕЙШЕГО ОСМЫСЛЕНИЯ.

6. В ЛИТЕРАТУРЕ СУДЕБНЫЕ ПОЛНОМОЧИЯ РОССИЙСКОГО МОНАРХА В КОНЦЕ XVII - ПЕРВОЙ ЧЕТВЕРТИ XVIII ВВ. БЕГЛО ОСВЕТИЛА ЕДИНСТВЕННО Т.Е. НОВИЦКАЯ (Т.Е. НОВИЦКАЯ, « ПРАВА И ПРЕИМУЩЕСТВА ИМПЕРАТОРА И ЧЛЕНОВ ИМПЕРАТОРСКОЙ ФАМИЛИИ», В А.А. ПРЕОБРАЖЕНСКОГО И Т.Е. НОВИЦКОЙ, РЕД., ЗАКОНОДАТЕЛЬСТВО ПЕТРА I, М. : ЮРИДИЧЕСКАЯ ЛИТЕРАТУРА, 1997, С. 4950).

7. ПОЛНОЕ СОБРАНИЕ ЗАКОНОВ РОССИЙСКОЙ ИМПЕРИИ С 1649 ГОДА, Т. 5, СПБ. : ТИПОГР. ІІ ОТДЕЛЕНИЯ Е. И. В. КАНЦЕЛЯРИИ, 1830, № 3316, с. 670.

8. ТАМ ЖЕ, Т. 7, № 4589, с. 361.

9. РГИА (РОССИЙСКИЙ ГОСУДАРТСВЕННЫЙ ИСТОРИЧЕСКИЙ АРХИВ), Ф. 1329, ОП. 1, Кн. 27, Л. 6868 оь.

10. РГАДА (РОССИЙСКИЙ ГОСУДАРСТВЕННЫЙ АРХИВ ДРЕВНИХ АКТОВ), Ф. 1451, КН. 13, Л. 49.

11. Н.А. ВОСКРЕСЕНСКИЙ, СОСТ., Б.И. СЫРОМЯТНИКОВ, РЕД., ЗАКОНОДАТЕЛЬНЫЕ АКТЫ ПЕТРА I, Т. 1, М.-Л. : ИЗД. АН СССР, 1945, с. 361-362, 377-379.

12. ТАМ ЖЕ, С. 289, 295, 378-379.

13. А.Г. МАНЬКОВ, РЕД., РОССИЙСКОЕ ЗАКОНОДАТЕЛЬСТВО Х-ХХ ВВ., Т. З, М.: ЮРИДИЧЕСКАЯ ЛИТЕРАТУРА, 1985, С. 104-105. М.А. ЧЕЛЬЦОВ-БЕБУТОВ ИНТЕРПРЕТИРОВАЛ ЗАПРЕТИТЕЛЬНУЮ НОРМУ НАЗВАННОЙ СТАТЬИ КАК ОТНОСИВШУЮСЯ ИСКЛЮЧИТЕЛЬНО К СУДЕБНЫМ ДЕЛАМ (М.А. ЧЕЛЬЦОВ-БЕБУТОВ, КУРС УГОЛОВНО-ПРОЦЕССУАЛЬНОГО ПРАВА: ОЧЕРКИ ПО ИСТОРИИ СУДА И 
УГОЛОВНОГО ПРОЦЕССА В РАБОВЛАДЕЛЬЧЕСКИХ, ФЕОДАЛЬНЫХ И БУРЖУАЗНЫХ ГОСУДАРСТВАХ, [2-Е ИЗД.], СПБ. : АЛЬФА ; РАВЕНА, 1995, С. 675). МЕЖДУ ТЕМ, УЖЕ В НАЧАЛЬНОЙ ФРАЗЕ СТ. 20 ГЛ. 10-Й ГОВОРИТСЯ О « ЛЮДЯХ, КОТОРЫМ... ДОВЕДЕТСЯ О СУДНЫХ СВОИХ И О ИНЫХ КАКИХ ДЕЛАХ БИТИ ЧЕЛОМ ГОСУДАРЮ». ИЗ ЭТОГО СО ВСЕЙ ОЧЕВИДНОСТЬЮ СЛЕДУЕТ, ЧТО В СТ. 20 ГЛ. 10-Й УЛОЖЕНИЯ 1649 Г. ОГРАНИЧИВАЛОСЬ ПРАВО ЧЕЛОБИТЬЯ КАК ТАКОВОГО, ВНЕ ЗАВИСИМОСТИ ОТ СОДЕРЖАНИЯ ЧЕЛОБИТНОЙ. ДЛЯ ПОЛНОТЫ КАРТИНЫ НЕОБХОДИМО ДОБАВИТЬ, ЧТО В СТ. 22 ГЛ. 2-Й УЛОЖЕНИЯ БЫЛО КОСВЕННО (ХОТЯ И НЕДВУСМЫСЛЕННО) ПОДТВЕРЖДЕНО ПРАВО « СЛУЖИЛЫХ И ИНЫХ ЧИНОВ ЛЮДЕЙ» НА ПОДАЧУ КОЛЛЕКТИВНОЙ ЧЕЛОБИТНОЙ С ЖАЛОБОЙ НА ВОЕВОД И ДРУГИХ ПРЕДСТАВИТЕЛЕЙ ГОСУДАРСТВЕННОГО АППАРАТА (РОССИЙСКОЕ ЗАКОНОДАТЕЛЬСТВО Х-ХХ ВВ., Т. 3, С. 89).

14. ПОЛНОЕ СОБРАНИЕ ЗАКОНОВ, Т. 4, № 1748, С. 3. БОЛЕЕ ИСПРАВНУЮ ПУБЛИКАЦИЮ ТЕКСТА УКАЗА СМ. : ДВОРЦОВЫЕ РАЗРЯДЫ, Т. 4, М., 1855, СТБ. 1112-1113.

15. В ЦЕЛОМ ЖЕ РАССМОТРЕННЫЙ ЗАКОН НЕСОМНЕННО ПРОДОЛЖИЛ ОТМЕЧЕННУЮ ВЫШЕ ЛИНИЮ УЛОЖЕНИЯ 1649 Г. НА ОГРАНИЧЕНИЕ ПРАВА ЧЕЛОБИТЬЯ.

16. ЗАКОНОДАТЕЛЬНЫЕ АКТЫ ПЕТРА I, С. 361.

17. ПОЛНОЕ СОБРАНИЕ ЗАКОНОВ, Т. 5, № 2756, с. 76.

18. ЗАКОНОДАТЕЛЬНЫЕ АКТЫ ПЕТРА I, С. 364. В ЛИТЕРАТУРЕ О НАЗВАННОМ ИМЕННОМ УКАЗЕ СМ. : Е.В. АНИСИМОВ, ДЫБА И КНУТ : ПОЛИТИЧЕСКИЙ СЫСК И РУССКОЕ ОБЩЕСТВО В XVIII ВЕКЕ, М. : НОВОЕ ЛИТЕРАТУРНОЕ ОБОЗРЕНИЕ, 1999, С. 19-20 ; Н.Н. ПОКРОВСКИЙ, « ЗАКОНОДАТЕЛЬНЫЕ ИСТОЧНИКИ О “СЛОВЕ И ДЕЛЕ ГОСУДАРЕВОМ”», ПУБЛИЦИСТИЧЕСКИЕ И ИСТОРИЧЕСКИЕ СОЧИНЕНИЯ ПЕРИОДА ФЕОДАЛИЗМА : СБОРНИК НАУЧНЫХ ТРУДОВ, НОВОСИБИРСК : НАУКА, 1989, с. 420-422 ; J. LeDonne, Absolutism and Ruling Class: The Formation of the Russian Political Order : 1700-1725, Oxford: Oxford University Press, 1991, c. 206-207.

19. ЗАКОНОДАТЕЛЬНЫЕ АКТЫ ПЕТРА I, С. 367.

20. ТАМ ЖЕ, С. 379.

21. РГАДА, Ф. 342, ОП. 1 , КН. 33, Ч. 1, Л. 12 ОБ.

22. ТАМ ЖЕ, Ф. 248, КН. 1887, Л. 129-130 ОБ., 308-309ОБ.

23. М.И. СЕМЕВСКИЙ, СЛОВО И ДЕЛО ! 1700-1725, СПБ. : ТИПОГР. В.С. БАЛАШЕВА, 1884, с. 50-51.

24. ЗАКОНОДАТЕЛЬНЫЕ АКТЫ ПЕТРА І, С. 377.

25. В.И. ВЕРЕТЕННИКОВ, ИСТОРИЯ ТАЙНОЙ КАНЦЕЛЯРИИ ПЕТРОВСКОГО ВРЕМЕНИ, ХАРЬКОВ : ПЕЧАТНОЕ ДЕЛО, 1910, С. 82-85, 109-130, 167 И ДР. ; Н.Б. ГОЛИКОВА, ПОЛИТИЧЕСКИЕ ПРОЦЕССЫ ПРИ ПЕТРЕ I : ПО МАТЕРИАЛАМ ПРЕОБРАЖЕНСКОГО ПРИКАЗА, М. : ИЗД. МГУ, 1957, с. 38-41, 44, 47-50, 52, 54, 61 И ДР. ; АНИСИМОВ, ДЫБА И КНУТ, с. 98-102, 110, 173-174 И ДР.

26. ОСВЕЩЕНИЕ ПРОЦЕССА АЛЕКСЕЯ ПЕТРОВИЧА В СОВРЕМЕННОЙ ЛИТЕРАТУРЕ СМ. : С.В. ЕФИМОВ, ПОЛИТИЧЕСКИЙ ПРОЦЕСС ПО ДЕЛУ ЦАРЕВИЧА АЛЕКСЕЯ ПЕТРОВИЧА: АВТОРЕФ. ДИС. ... КАНД. ИСТ. НАУК, СПБ., 1997 ; Р. Bushkovitch, Peter the Great: The Struggle for Power : 1671-1725, Cambridge : Cambridge University Press, 2001, с. 383-425. КАК ИЗВЕСТНО, В ХОДЕ ПРОЦЕССА ДЕЛО ДОШЛО И ДО ЛИЧНОГО УЧАСТИЯ ПЕТРА І В ПЫТКАХ ЦАРЕВИЧА (ОБ ЭТОМ МРАЧНОМ СЮЖЕТЕ СМ., В ПЕРВУЮ ОЧЕРЕДЬ : Г.В. ЕСИПОВ, «КАБАЧОК “МАРТЫШКА” : ЭПИЗОД 1718-1719 ГГ.», РУССКИЙ ВЕСТНИК, № 11, 1860, с. 115-126).

27. РГАДА, Ф. 6, № 55, Л. 1-2ОБ., 9-9ОБ., 14 ОБ.-15, 19-19ОБ. В ЛИТЕРАТУРЕ О ПРОЦЕССЕ НАД В.В. ДОЛГОРУКОВЫМ 1718 Г. НАИБОЛЕЕ ПОДРОБНО СМ. : Н.Г. УСТРЯЛОВ, ИСТОРИЯ ЦАРСТВОВАНИЯ ПЕТРА ВЕЛИКОГО, Т. 6, СПБ. : ТИПОГР. ІІ ОТДЕЛЕНИЯ Е. И. В. КАНЦЕЛЯРИИ, 1859, С. 195-201.

28. РГИА, Ф. 1329, ОП. 1, КН. 27, л. 9, 11, 18-19, 28, 40, 46.

29. ТАМ ЖЕ, Л. 69-70ОБ.

30. РГИА, Ф. 1329, ОП. 1 , КН. 27, Л. 286ОБ. ПИСЬМО БЫЛО ИЗЪЯТО В АМСТЕРДАМСКОЙ КОНТОРЕ О.А. СОЛОВЬЕВА В 1717 Г. В ХОДЕ ОБЫСКА, ПРОИЗВЕДЕННОГО В РАМКАХ РАССЛЕДОВАНИЯ « АРХАНГЕЛЬСКОГО ДЕЛА». 
31. ПОХОДНЫЙ ЖУРНАЛ 1714 ГОДА, СПБ., 1854, с. 144-145, 148. В ЛИТЕРАТУРЕ О « ПОДРЯДНОМ ДЕЛЕ» СМ. : Н.И. ПАВЛЕНКО, ПЕТР ВЕЛИКИЙ, М. : МЫСЛЬ, 1994, с. 501-503; Bushkovitch, Peter the Great, c. 322-334.

32. РГАДА, Ф. 248, КН. 300, Л. 10-11, 14, 58 И ДР. ПУБЛИКАЦИЮ (В ВИДЕ ПЕРЕСКАЗА) ОСНОВНОЙ ЧАСТИ МАТЕРИАЛОВ СУДЕБНОГО ПРОИЗВОДСТВА ПО ДЕЛУ П.П.ШАФИРОВА И Г.Г. СКОРНЯКОВА-ПИСАРЕВА СМ. : П.И. ИВАНОВ, « СУДНОЕ ДЕЛО НАД ДЕЙСТВИТЕЛЬНЫМ ТАЙНЫМ СОВЕТНИКОМ БАРОНОМ ШАФИРОВЫМ И ОБЕР-ПРОКУРОРОМ СЕНАТА СКОРНЯКОВЫМ-ПИСАРЕВЫМ», ЖУРНАЛ МИНИСТЕРСТВА ЮСТИЦИИ, Т. 1, ч. 3, 1859, с. 8-62.

33. РГАДА, Ф. 248, КН. 300, л. 72.

34. ТАМ ЖЕ, КН. 273, Л. 428, 633, 635. СТОИТ ЗАМЕТИТЬ, ЧТО ПЕРВЫЙ РАЗ ПЕТР I САНКЦИОНИРОВАЛ ПРИМЕНЕНИЕ К А.Я. НЕСТЕРОВУ ПЫТКИ, КОГДА ЕЩЕ НАХОДИЛСЯ В ПЕРСИДСКОМ ПОХОДЕ - В ПИСЬМЕ К ГЛАВЕ СЛЕДСТВЕННОЙ КАНЦЕЛЯРИИ ГЕНЕРАЛ-ПРОКУРОРУ П.И. ЯГУЖИНСКОМУ ОТ 15 ОКТЯБРЯ 1722 Г. ИЗ АСТРАХАНИ (РГИА, Ф. 1329, ОП. 1, КН. 28, Л. 3). СООБРАЗНО С ВЫСОЧАЙШИМ ДОЗВОЛЕНИЕМ, « ЗАСТЕНОК» АЛЕКСЕЮ НЕСТЕРОВУ БЫЛ НАЗНАЧЕН ПОСТАНОВЛЕНИЕМ СЛЕДСТВЕННОЙ КАНЦЕЛЯРИИ ОТ 8 НОЯБРЯ 1722 Г. (РГАДА, Ф. 248, КН. 273, Л. 226-230). ОДНАКО, КАК ЯВСТВУЕТ ИЗ МАТЕРИАЛОВ ДЕЛА, НАМЕЧЕННЫЙ ДОПРОС ПОДСЛЕДСТВЕННОГО ПОД ПЫТКОЙ ТАК И НЕ СОСТОЯЛСЯ, БУДУЧИ ОТЛОЖЕН ДО ВОЗВРАЩЕНИЯ ИМПЕРАТОРА ИЗ ПОХОДА.

35. РГАДА, Ф. 9, ОТД. 1, КН. 58, Л. 572.

36. ТАМ ЖЕ, Ф. 11, № 371, Л. 5-8ОБ.

37. ЗАКОНОДАТЕЛЬНЫЕ АКТЫ ПЕТРА I, с. 220. ЕСЛИ ПРИ ЭТОМ ВСПОМНИТЬ, ЧТО РЕВИЗИОН-КОЛЛЕГИЯ ОТВЕЧАЛА ЗА ФИНАНСОВЫЙ КОНТРОЛЬ В СТРАНЕ, ТО ОБРИСОВАННАЯ СИТУАЦИЯ ПРИОБРЕТАЕТ И ВОВСЕ АБСУРДНЫЙ ОТТЕНОК.

38. ПОДРОБНЕЕ ОБ ЭТОМ ПРОЦЕССЕ СМ.: В.И.САМОЙЛОВ, «О ПЕРВЫХ УЗНИКАХ ПЕТРОПАВЛОВСКОЙ КРЕПОСТИ», СОВЕТСКОЕ ГОСУДАРСТВО И ПРАВО, № 10, 1956, с. 119-121.

39. АНИСИМОВ, ДЫБА И КНУТ, с. 110.

40. РГАДА, Ф. 248, КН. 1886, Л. 154ОБ.-156.

41. ТАМ ЖЕ, КН. 273, Л. 440.

42. ВЕРЕТЕННИКОВ, ИСТОРИЯ ТАЙНОЙ КАНЦЕЛЯРИИ ПЕТРОВСКОГО ВРЕМЕНИ, С. 109-110.

43. РГАДА, Ф. 1451, КН. 13, Л. 52 ; ТАМ ЖЕ, Ф. 248, КН. 1888, Л. 311 ОБ.

44. РГИА, Ф. 1329, ОП. 1, КН. 28, Л. 13-25ОБ., 37-48.

45. ЗАКОНОДАТЕЛЬНЫЕ АКТЫ ПЕТРА I, С. 259.

46. ПОХОДНЫЙ ЖУРНАЛ 1724 ГОДА, СПБ., 1854, с. 34-36.

47. ГОЛИКОВА, ПОЛИТИЧЕСКИЕ ПРОЦЕССЫ ПРИ ПЕТРЕ I, С. 39.

48. ТАМ ЖЕ, С. $47,50$.

49. ТАM ЖЕ, С. 44.

50. ГОДОВОЕ ЖАЛОВАНЬЕ КОМИССАРА В САНКТ-ПЕТЕРБУРГЕ, СОГЛАСНО ИМЕННОМУ УКАЗУ ОТ 28 ЯНВАРЯ 1715 Г., СОСТАВЛЯЛО 120 РУБЛЕЙ (ПОЛНОЕ СОБРАНИЕ ЗАКОНОВ, Т. 5, № 2879, с. 139).

51. РГАДА, Ф. 285, ОП. 1, КН. 5947, № 185, Л. 9.

52. ГОЛИКОВА, ПОЛИТИЧЕСКИЕ ПРОЦЕССЫ ПРИ ПЕТРЕ I, С. 54.

53. РГВИА (РОССИЙСКИЙ ГОСУДАРСТВЕННЫЙ ВОЕННО-ИСТОРИЧЕСКИЙ АРХИВ), Ф. 2583, ОП. 1, № 5, Л. 46-46ОБ. О ДЕЛЕ Н.Т. РЖЕВСКОГО СМ. : Д.О. СЕРОВ, « ИЗ ИСТОРИИ ВОЕННОЙ ЮСТИЦИИ ПЕТРОВСКОГО ВРЕМЕНИ : ПРОЦЕСС Н.Т. РЖЕВСКОГО (1710-1714)», КРОВЬ. ПОРОХ. ЛАВРЫ. ВОЙНЫ РОССИИ В ЭПОХУ БАРОККО (1700-1762) : СБОРНИК МАТЕР. ВСЕРОС. НАУЧН. КОНФ., ВЫП. 2, СПБ. : ВИМАИВИВС, 2002, с. 41-43.

54. РГАДА, Ф. 1451, КН. 10, Л. 40.

55. ТАМ ЖЕ, Ф. 285, ОП. 1, КН. 5947, № 185, Л. 8-8ОБ.

56. САМОЙЛОВ, « О ПЕРВЫХ УЗНИКАХ ПЕТРОПАВЛОВСКОЙ КРЕПОСТИ», С. 121. 
57. РГАДА, Ф. 285, ОП. 1, КН. 5947, № 185, Л. 8ОБ.-9.

58. РГАДА, Ф. 248, КН. 300, Л. 263-266.

59. ТАМ ЖЕ, КН. 30, Л. 185.

60. ТАМ ЖЕ, КН. 273, Л. 701-701ОБ.

61. ТАМ ЖЕ, КН. 274, Л. 1443, 1444 ОБ.

62. РГИА, Ф. 1329 , ОП. 1 , КН. 17 , Л. 96ОБ.; РГАДА, Ф. 248, КН. 273, Л. 691 . ПОДРОБНЕЕ СМ. : Д.О.СЕРОВ, «ВЫСШИЕ АДМИНИСТРАТОРЫ ПОД СУДОМ ПЕТРА І (ИЗ ИСТОРИИ УГОЛОВНОЙ ЮСТИЦИИ РОССИИ ПЕРВОЙ ЧЕТВЕРТИ XVIII В.)», ИЗВЕСТИЯ УРАЛЬСКОГО ГОСУДАРСТВЕННОГО УНИВЕРСИТЕТА, № 39, 2005, С. 47-63.

63. ПЕТР І ПРИГОВОРИЛ Г.П. ЧЕРНЫШЕВА К ШТРАФУ В РАЗМЕРЕ ТРОЕКРАТНОГО ЖАЛОВАНЬЯ СОЛДАТ И ПОЛОВИННОГО ДЕНЕЖНОГО СОДЕРЖАНИЯ КАТОРЖНИКОВ ЗА ТО ВРЕМЯ, КОГДА ОНИ НЕЗАКОННО ИСПОЛЬЗОВАЛИСЬ НА СТРОИТЕЛЬСТВЕ ГЕНЕРАЛЬСКОГО ДОМА - ЧТО И СОСТАВИЛО УКАЗАННУЮ ВЫШЕ СУММУ В 372 РУБЛЯ.

64. РГАДА, Ф. 248, КН. 300, Л. 272.

65. ГОЛИКОВА, ПОЛИТИЧЕСКИЕ ПРОЦЕССЫ ПРИ ПЕТРЕ I, С. 54.

66. РГАДА, Ф. 248, КН. 273, Л. 827-827ОБ.

67. Н.Ф. ДУБРОВИН, РЕД., СБОРНИК ИМПЕРАТОРСКОГО РУССКОГО ИСТОРИЧЕСКОГО ОБЩЕСТВА, Т. 101, СПБ. : ТИПОГР. И.Н. СКОРОХОДОВА, 1898, с. 200, 208-209.

68. ИСТОРИЯ ВЗАИМООТНОШЕНИЙ В. МОНСА И ЕКАТЕРИНЫ АЛЕКСЕЕВНЫ, А ТАКЖЕ ВНЕШНИЕ ОБСТОЯТЕЛЬСТВА РАЗОБЛАЧЕНИЯ И ОСУЖДЕНИЯ КАМЕРГЕРА ДЕТАЛЬНО ОСВЕЩЕНЫ М.И. СЕМЕВСКИМ (М.И. СЕМЕВСКИЙ, ЦАРИЦА КАТЕРИНА АЛЕКСЕЕВНА, АННА И ВИЛЛИМ МОНС : 16921724, 2-Е ИЗД., СПБ. : ТИПОГР. В.С. БАЛАШЕВА, 1884, с. 106-129, 170-226). С ИСТОРИКО-ПРАВОВОЙ СТОРОНЫ ПРОЦЕСС НАД В. МОНСОМ МИХАИЛ СЕМЕВСКИЙ, ПРАВДА, НЕ АНАЛИЗИРОВАЛ.

69. РГАДА, Ф.6, № 157, КН. 1, Л.578-579. ПРЕСТУПНЫЕ ДЕЯНИЯ В. МОНСА БЫЛИ КВАЛИФИЦИРОВАНЫ СУДОМ ПО СТ. 2 ГЛ. 50-Й ГЕНЕРАЛЬНОГО РЕГЛАМЕНТА 1720 Г. И ПО СТ. 1 ИМЕННОГО УКАЗА ОТ 25 ОКТЯБРЯ 1723 Г. ОБ УСИЛЕНИИ ОТВЕТСТВЕННОСТИ ЗА ПРЕСТУПЛЕНИЯ ПРОТИВ ИНТЕРЕСОВ СЛУЖБЫ.

70. СТОИТ ЗАМЕТИТЬ, ЧТО ПЕТР І ОТНЮДЬ НЕ ЯВИЛСЯ НОВАТОРОМ В ПРИМЕНЕНИИ ПОДОБНОЙ ДОПОЛНИТЕЛЬНОЙ САНКЦИИ. О СЛОЖИВШЕЙСЯ В XVII В. (И ПРОТЯНУВШЕЙСЯ ЗАТЕМ ЧЕРЕЗ ВЕСЬ XVIII В.) РОССИЙСКОЙ ТРАДИЦИИ ФАКУЛЬТАТИВНОГО НЕПОГРЕБЕНИЯ ТЕЛ КАЗНЕННЫХ СМ.: АНИСИМОВ, ДЫБА И КНУТ, с. 584-588.

71. ПИСЬМА И БУМАГИ ИМПЕРАТОРА ПЕТРА ВЕЛИКОГО, Т. 12, ВЫП. 1, М. : НАУКА, 1975, с. 89, 365-366.

72. УСТРЯЛОВ, ИСТОРИЯ ПЕТРА ВЕЛИКОГО, Т. 6, С. 617.

73. 25 НОЯБРЯ 1721 Г. ПЕТР І ДОПОЛНИТЕЛЬНО РАСПОРЯДИЛСЯ ПЕРЕВЕСИТЬ ТЕЛО М.П. ГАГАРИНА НА ОСОБО ИЗГОТОВЛЕННУЮ ЦЕПЬ (РГАДА, Ф. 1451, КН. 16, Л. 97).

74. СЕМЕВСКИЙ, ЦАРИЦА КАТЕРИНА АЛЕКСЕЕВНА, АННА И ВИЛЛИМ МОНС, С. 224.

75. РГАДА, Ф. 248, КН. 703, Л. 134.

76. ТАМ ЖЕ, Ф. 9, ОТД. 2 , КН. 56, Л. 229. ХАРАКТЕРНО, ЧТО К ТОМУ ВРЕМЕНИ БЫЛО ЗАВЕРШЕНО РАССЛЕДОВАНИЕ ТОЛЬКО 12 ИЗ 27 ЭПИЗОДОВ, ПО КОТОРЫМ ОБВИНЯЛСЯ А.А. КУРБАТОВ, И САМ ОН НАХОДИЛСЯ НЕ ПОД СТРАЖЕЙ, А ПОД ПОДПИСКОЙ О НЕВЫЕЗДЕ (ТАМ ЖЕ, Л. 231-231 ОБ. ; ТАМ ЖЕ, Ф. 248, КН. 218, Л. 206).

77. РГАДА, Ф. 248, КН. 1935, Л. 241ОБ. ; ТАМ ЖЕ, ОП. 110, № 160, Л. 60.

78. ТАМ ЖЕ, КН. 1888, Л. 177ОБ. ВЕРНУВШИСЬ ИЗ СИБИРИ, Н.Т. РЖЕВСКИЙ СУМЕЛ (НАПОМНИВ О СВОИХ БОЕВЫХ ЗАСЛУГАХ) ДОБИТЬСЯ ПОЛНОЙ РЕАБИЛИТАЦИИ, КАКОВАЯ ПОСЛЕДОВАЛА, ПО ИМЕННОМУ УКАЗУ ОТ 11 МАЯ 1722 Г. (ТАМ ЖЕ, Ф. 1451, КН. 13, Л. 374).

79. П.И. БАРАНОВ, СОСТ., АРХИВ ПРАВИТЕЛЬСТВУЮЩЕГО СЕНАТА, Т. 1, СПБ.: ТИПОГР. ПРАВИТ. СЕНАТА, 1872, с. 129. 23 ДЕКАБРЯ 1724Г. ИМПЕРАТОР ТАКЖЕ ПРОИЗВЕЛ БЫВШЕГО ГЕНЕРАЛ-ЛЕЙТЕНАНТА В.В. ДОЛГОРУКОВА В ЧИН ПОЛКОВНИКА (ТАМ ЖЕ, С. 137). 
80. РГАДА, Ф. 248, КН. 1922, Л. 77ОБ. ; ТАМ ЖЕ, КН. 300, Л. 331-332ОБ. 15 ЯНВАРЯ 1724 Г. ПЕТР І ДОПОЛНИТЕЛЬНО ОСВОБОДИЛ ОБОИХ СЕНАТОРОВ ОТ УПЛАТЫ НЕ ВНЕСЕННОЙ К ТОМУ ВРЕМЕНИ СУММЫ ШТРАФА (ТАМ ЖЕ, Л. 345).

\section{RÉSUMÉS}

L'article étudie les activités du tsar et empereur Pierre $\mathrm{I}^{\mathrm{er}}$ dans le domaine de la justice. Pour la première fois dans l'historiographie, les actes législatifs du premier quart du XVIII ${ }^{\mathrm{e}}$ siècle destinés à réguler l'activité judiciaire du monarque russe sont analysés de façon systématique et c'est sur la base de très nombreux documents d'archives que l'auteur révèle la participation personnelle de Pierre $\mathrm{I}^{\mathrm{er}}$ à des procès. Il met en évidence l'intérêt manifeste du premier empereur de la Russie pour le fonctionnement de la justice, en premier lieu pour les procédures judiciaires relevant du pénal, (crimes d'État et délits de prévarication, notamment) et s'attache à proposer une analyse détaillée des décisions de Pierre $\mathrm{I}^{\mathrm{er}}$ concernant la confirmation des peines ou leur rémission.

The article reviews the activities of Tsar and Emperor Peter the Great in the judicial sphere. For the first time in historiography, the author systematically analyzes Russian legislative acts of the first quarter of the eighteenth century regulating the monarch's participation in judicial proceedings. Based on a wide range of archival sources, it describes Peter the Great's personal involvement in litigations. It shows that the first Russian emperor paid considerable attention to the problems of justice and that he had special interest in criminal proceedings (particularly in cases of crimes against the state and maladministration). Particular attention is paid to the analysis of Peter the Great's decisions concerning approval or remission of sentences.

\section{AUTEUR}

\section{DMITRIJ O. SEROV}

НОВОСИБИРСКИЙ ГОСУДАРСТВЕННЫЙ УНИВЕРСИТЕТ ЭКОНОМИКИ И УПРАВЛЕНИЯ (Novosibirsk State University of Economics and Management). serov1313@mail.ru 